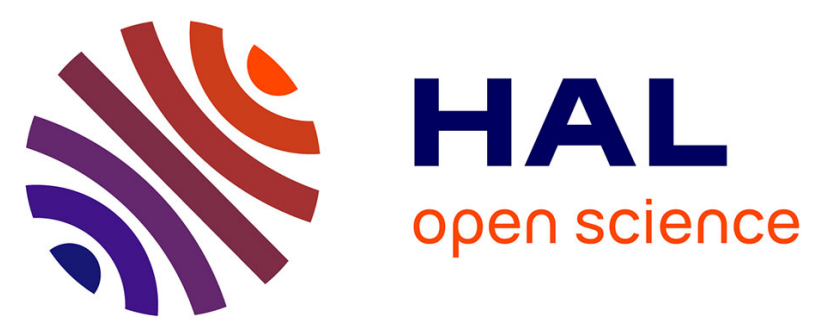

\title{
Laser line illumination scheme allowing the reduction of background signal and the correction of absorption heterogeneities effects for fluorescence reflectance imaging
}

Frédéric Fantoni, Lionel Hervé, Vincent Poher, Sylvain Gioux, Jerome I. Mars, Jean-Marc Dinten

\section{To cite this version:}

Frédéric Fantoni, Lionel Hervé, Vincent Poher, Sylvain Gioux, Jerome I. Mars, et al.. Laser line illumination scheme allowing the reduction of background signal and the correction of absorption heterogeneities effects for fluorescence reflectance imaging. Journal of Biomedical Optics, 2015, 20 (10), pp.11. 10.1117/1.JBO.20.10.106003 . hal-01253488

\author{
HAL Id: hal-01253488 \\ https://hal.science/hal-01253488
}

Submitted on 10 Jan 2016

HAL is a multi-disciplinary open access archive for the deposit and dissemination of scientific research documents, whether they are published or not. The documents may come from teaching and research institutions in France or abroad, or from public or private research centers.
L'archive ouverte pluridisciplinaire HAL, est destinée au dépôt et à la diffusion de documents scientifiques de niveau recherche, publiés ou non, émanant des établissements d'enseignement et de recherche français ou étrangers, des laboratoires publics ou privés. 


\title{
Laser line illumination scheme allowing the reduction of background signal and the correction of absorption heterogeneities effects for fluorescence reflectance imaging
}

\author{
Frédéric Fantoni, ${ }^{a}$ Lionel Hervé, ${ }^{a}$ Vincent Poher, ${ }^{a}$ Sylvain Gioux, ${ }^{b}$ Jérôme I. Mars, ${ }^{c}$ \\ Jean-Marc Dinten a \\ aUniv. Grenoble Alpes, F-38000 Grenoble, France \\ CEA, LETI, MINATEC Campus, F-38054 Grenoble, France

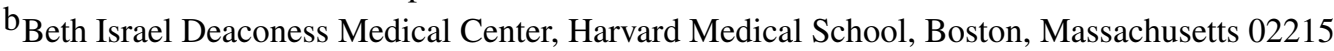 \\ ${ }^{\mathrm{c}}$ Univ. Grenoble Alpes, Gipsa-Lab, F-38000 Grenoble, France \\ CNRS, Gipsa-Lab, F-38000 Grenoble, France
}

\begin{abstract}
Intraoperative fluorescence imaging in reflectance geometry is an attractive imaging modality as it allows to noninvasively monitor the fluorescence targeted tumors located below the tissue surface. Some drawbacks of this technique are the background fluorescence decreasing the contrast and absorption heterogeneities leading to misinterpretations concerning fluorescence concentrations. We propose a correction technique based on a laser line scanning illumination scheme. We scan the medium with the laser line and acquire at each position of the line both fluorescence and excitation images. We then use the finding that there is a relationship between the excitation intensity profile and the background fluorescence one to predict the amount of signal to subtract to the fluorescence images to get a better contrast. As the light absorption information is contained both in fluorescence and excitation images, this method also permits us to correct the effects of absorption heterogeneities. This technique has been validated on simulations and experimentally. Fluorescent inclusions are observed in several configurations at depths ranging from $1 \mathrm{~mm}$ to $1 \mathrm{~cm}$. Results obtained with this technique are compared to those obtained with a classical wide-field detection scheme for the contrast enhancement and to the fluorescence by excitation ratio approach for the absorption correction.
\end{abstract}

Keywords: Medical and biological imaging, Imaging systems, Illumination design, Image reconstruction techniques.

Address all correspondence to: Lionel Hervé, CEA LETI, DTBS-STD-LISA, 17 rue des Martyrs, 38054 Grenoble France. Tel: +33 438786 059; E-mail: lionel.herve@cea.fr

\section{Introduction}

The interest to molecular imaging has increased for the past few years, ${ }^{1-4}$ due to the recent availability of fluorochromes which allow, for example, the study of gene expression, protein function and interactions, and a large number of cellular processes in a minimally invasive way.

Molecular imaging presents several advantages compared to other forms of tissue imaging: it offers good sensitivity when the observed objects are close to the surface, is generally fast (acquisition times typically range from a fraction of seconds to minutes) and the instruments can be easy to implement, low cost and compact. 
Still, as the signal to background ratio is a major factor in fluorescence imaging, this technique suffers from an important limitation that is linked to the background signal caused by several sources such as excitation leaks, non-specific fluorescence from injected fluorophores and/or fluorescence from superficial layers. Contrary to the visible spectrum, ${ }^{5}$ endogenous sources of fluorescence emit a signal that can be considered negligible in terms of intensity compared to the signal of near-infrared (NIR) fluorescence probes in the NIR spectrum, allowing the contrast to be much better as long as the target studied is not located too deeply inside the tissue. However, as the amount of fluorophore bound on a target is generally low (the concentration being of the nanomolar order), ${ }^{6}$ the natural fluorescence of tissues coupled to the other parasite signals (excitation leaks, non-specific fluorescence, or fluorescence from the $\operatorname{diet}^{7-9}$ ) can then become an obstacle and lead to a limited depth of study: the background signal, even if relatively weak, remains the same while the fluorescence of interest decreases exponentially with depth.

There are several methods used to suppress the effects of background signal, and most of them are spectral-based methods which consist in acquiring images with several wavelengths to obtain a spectral data cube of the object studied. It is then possible to use source separation techniques to obtain the fluorescence contribution from the parasite signals and then suppress it.

For example, the Carestream Health system ${ }^{10}$ uses excitation wavelengths between 390 and $770 \mathrm{~nm}$ and emission wavelengths between 440 and $830 \mathrm{~nm}$. The different excitation and emission fluorescence spectra are then fitted to a model to identify the contribution of the background fluorescence. The Maestro system from the Cambridge Resarch and Instrumentation team ${ }^{11}$ is based on a similar method. It uses several excitation and emission wavelengths and the fact that the fluorescence spectra from the different fluorophores (endogenous and exogenous) add linearly. We can 
also cite the IVIS system from the Caliper Life Sciences group. ${ }^{12}$ In this case, the measurements at the different excitation and emission wavelengths are decomposed in components matrices $C$ and spectra matrices $S$. The algorithm used then allows the separation between the autofluorescence spectrum and the spectra from the different exogenous fluorophores. It is however necessary to initialize the matrices $C$ and $S$ and to know the number of sources observed.

It is also possible to take advantage of the fact that the autofluorescence can be excited with a larger range of wavelengths compared to the usual exogenous fluorophores. Two wavelengths can be used, one exciting both the autofluorescence and the fluorescence of interest, the other exciting only the autofluorescence. ${ }^{13-15}$ The autofluorescence spectrum being the same for the two wavelengths, it is then possible to subtract the autofluorescence contribution.

The other background fluorescence reduction methods are based on tissue photobleaching ${ }^{16}$ (by illuminating them before the observation of the fluorescence of interest, the background signal can be strongly reduced), or on the properties of fluorophores (larger Stokes shifts ${ }^{17,18}$ or lifetimes $^{19}$ ).

Contrary to these techniques, we propose a background fluorescence reduction method that relies on the illumination and detection geometries. Classical fluorescence reflectance imaging is usually performed with a wide-field detection scheme (referred as WF-FRI). The method we propose consists in illuminating the medium with a laser line that scans the area of study. In our previous paper, ${ }^{20}$ we took advantage of this type of illumination to enhance the contrast and resolution of fluorescence reflectance imaging. The method proposed here consists in acquiring fluorescence and excitation images at each position of the illumination line. By using the relationship between the excitation intensity profile and the background fluorescence one, we are able to predict the amount of signal to subtract to the fluorescence images to get a better contrast. We will 
also show how this method allows us to enhance the resolution and correct some of the effects of absorption heterogeneities, leading to a better accuracy for the detection.

In the first part, we will describe the setup used for the study and explain the processing performed on the stack of images acquired. We will then present results on different cases (a phantom with a single fluorescent inclusion, a fluorescent resolution target, and two phantoms with varying absorptions heterogeneities) and show that this method improves both the contrast and the resolution and is robust against some of the effects of absorption heterogeneities.

\section{Materials and methods}

\subsection{Experimental setup}
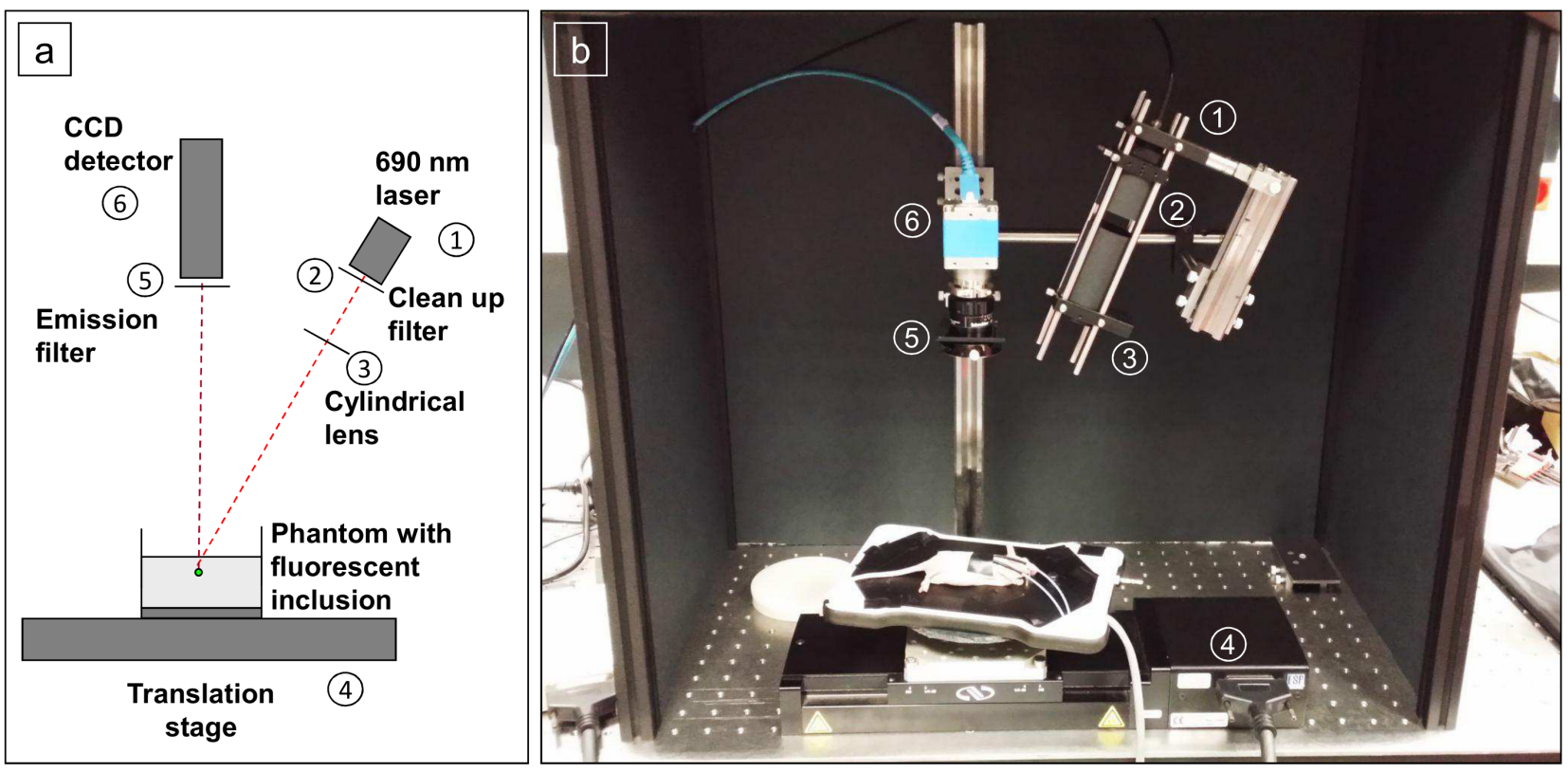

Fig 1 Optical setup used during the study (schematic (a) and platform (b))

Most of the elements of the optical setup (illustrated on Fig. 1) used for this study are classical and based on a common setup for reflectance molecular imaging. The light source (noted 1 on Fig. 1 is a $690 \mathrm{~nm}$ fibered laser (Intense HPD model 7404) which illuminates a tissue-like liquid 
phantom. A 690/10 nm clean up filter was used (noted 2 on Fig. 1). Fluorescence images are acquired with a CCD camera (PCO Pixelfly VGA, 640x480 pixels images, noted 6 on Fig. 1) for each position of the object which rests on a motorized translation stage (noted 4 on Fig. 1). A cylindrical lens (noted 3 on Fig. 1) is used to focus the laser at $45^{\circ}$ on the phantom along a line of length $4 \mathrm{~cm}$ and width $1 \mathrm{~mm}$ which sets the translation steps at $1 \mathrm{~mm}$ to fully illuminate the phantom.

For this study, the excitation line is static and the object observed is translated. While this is not a problem for this proof of principle where only phantoms were studied, a better implementation of the setup would keep the object observed static and we would move the excitation line. This would indeed be more practical, in particular for in vivo imaging.

The optical setup was optimized to minimize the amount of excitation bleed-through by using proper filters. A fluorescence filter (Semrock Razoredge $808 \mathrm{~nm}$ long pass filter, noted 5 on Fig. 1) is in front of the camera to stop all excitation photons and so as to detect a fluorescence signal. The laser power is $15 \mathrm{~mW}$.

For all experiments presented here, the stack of fluorescence images was first acquired and then the stack of excitation images was acquired. For the acquisition of excitation images, the fluorescence filter is replaced manually with a neutral density filter. The fact that we switch filters manually does not cause any significant distortion between fluorescence and excitation images for these experiments where only still optical phantoms are observed. To image living subjects, we should however be able to acquire fluorescence and excitation images at the same time to avoid distortions caused by movements of the subject.

For the contrast enhancement experiments, 80 images were acquired for each depth considered, 
with integration times ranging from $30 \mathrm{~ms}$ at $1 \mathrm{~mm}$ to $3 \mathrm{~s}$ at $1 \mathrm{~cm}$. The acquisition was automated so that one second passed between each acquisition to ensure that the liquid phantom was completely still and did not move because of the translation. This leads to total scanning times ranging from $82.4 \mathrm{~s}$ at $1 \mathrm{~mm}$ to $320 \mathrm{~s}$ at $1 \mathrm{~cm}$. No significant photobleaching was observed for any of the experiments.

For the resolution enhancement experiments, we used $100 \mu \mathrm{m}$ translation steps to be able to use thinner detection stripes. 400 images were acquired for each depth considered, with integration times ranging from $30 \mathrm{~ms}$ at $1 \mathrm{~mm}$ to $250 \mathrm{~ms}$ at $4 \mathrm{~mm}$. This leads to total scanning times ranging from $412 \mathrm{~s}$ at $1 \mathrm{~mm}$ to $500 \mathrm{~s}$ at $4 \mathrm{~mm}$. No significant photobleaching was observed for any of the experiments.

All the liquid phantoms used in this study were made with the same recipe to obtain tissuelike optical properties with an absorption coefficient $\mu_{a}=0.05 \mathrm{~cm}^{-1}$ and a reduced scattering coefficient $\mu_{s}^{\prime}=10 \mathrm{~cm}^{-1}$. The fluorescent inclusions are $1 \mathrm{~mm}$ diameter tubes containing 3 $\mu \mathrm{M}$ of Indocyanine Green encapsulated in lipid nanoparticles $\left(\mathrm{LNP}-\mathrm{ICG}^{21}\right.$ ) diluted in the same preparation as the phantoms to match background optical properties. We added different amounts of LNP-ICG to the phantom preparation to obtain different ratios of fluorescence to background fluorescence. The LNP-ICG absorption and fluorescence emission spectra are presented on Fig. 2.d.

Four different types of phantoms were used for this study:

- a single inclusion located at different depths ranging from $1 \mathrm{~mm}$ to $1 \mathrm{~cm}$,

- a fluorescent resolution target depicted in Fig. 2.a. This target is made of plexiglass pieces (which fluorescence emission spectrum is given on the right of Fig. 2.a) embedded in a 

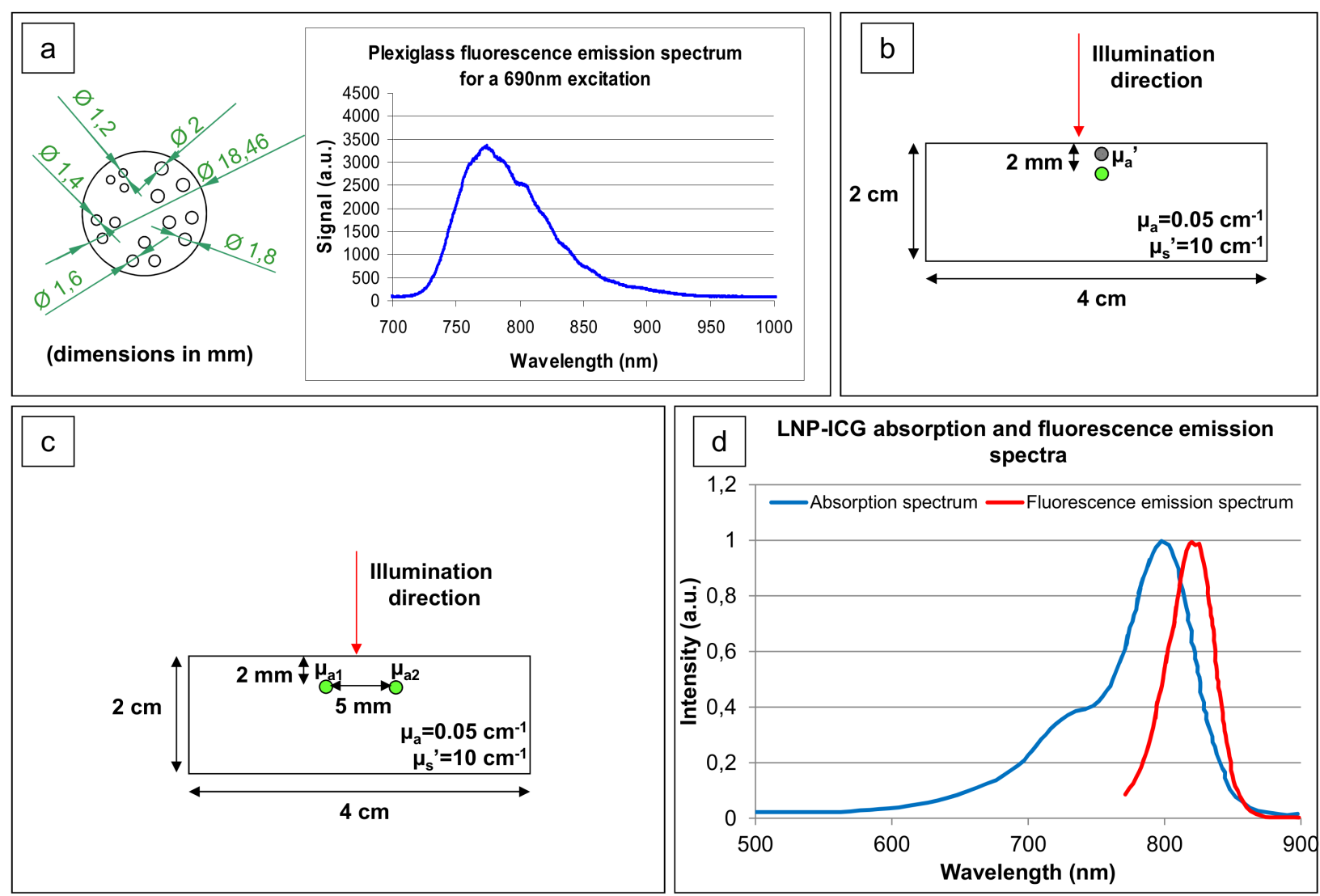

Fig 2 (a): Fluorescence target used for the resolution study; (b),(c): Phantoms used for the absorption correction study; (d): LNP-ICG fluorescence excitation and emission spectra.

circular phantom made of polyester resin with optical properties matching those of the liquid phantoms. The target is then immerged in a liquid phantom at depths ranging from $1 \mathrm{~mm}$ to $3 \mathrm{~mm}$ to test the improvements in resolution with depth,

- a single inclusion located $2 \mathrm{~mm}$ below the surface and under a $1 \mathrm{~mm}$-diameter absorption heterogeneity with an absorption coefficient $\mu_{a}^{\prime}$ ten times higher than the surrounding absorption coefficient (depicted on Fig. 2.b),

- two inclusions located at the same depths but with different absorption coefficient (depicted on Fig. 2.c, this will be described more precisely in the absorption correction part of this paper $(\S 3.3))$. 


\subsection{Image processing methods}

\subsubsection{Wide-field image: WF-FRI/Comparison with classical FRI}

To be able to qualitatively compare the proposed LS-FRI methods to the classical FRI, we must first obtain the equivalent of a wide-field illumination image. As the illumination obtained with an expanded beam is the same as the one obtained from the sum of several illuminations covering the same area, we can obtain a WF-FRI image by summing the stack of images at all positions with a shift depending on the translation step of the object.

Details on the comparison between this way of obtaining the WF-FRI image and a real widefield illumination can be found in our previous article ${ }^{20}$.

\subsubsection{Method proposed}

The processing method we will present relies on the assumption that there is a relationship between the excitation and the autofluorescence signals defined as following 22 :

$$
\frac{A(r)}{E(r)}=\beta . r
$$

where $E$ is the excitation, $A$ is the autofluorescence, $r$ is the distance and $\beta$ is a proportionality coefficient which depends on the optical properties of the medium and the background fluroescence yield. The geometry and intensity profiles are depicted on Fig. 3.

If the assumption is true, knowing the excitation profile could give us an insight on the autofluorescence profile. Simulations and experimental validations were performed to test this relationship, with simulations parameters for the optical properties chosen to match the experimental conditions. 


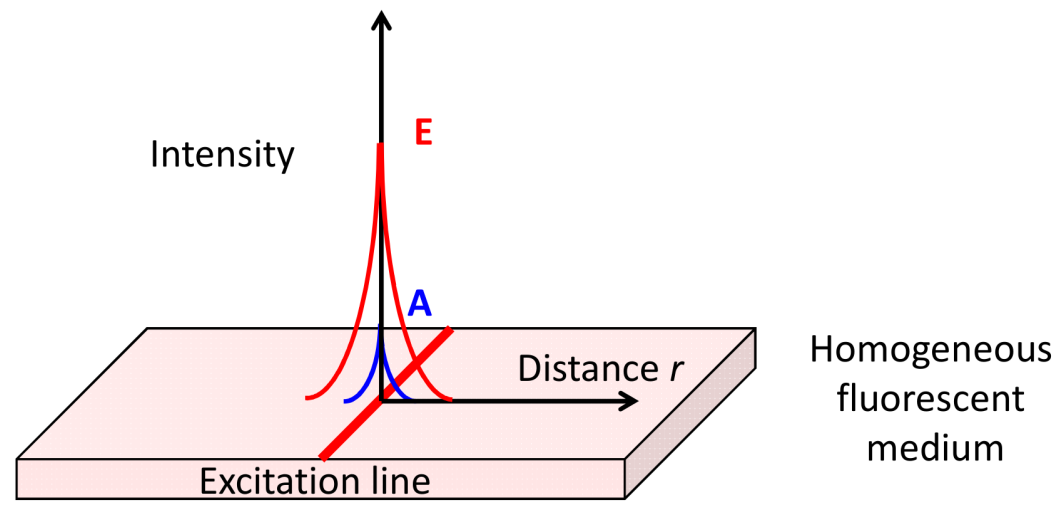

Fig 3 Schematic of the geometry studied: a homogeneous fluorescent medium is considered and we look at the excitation (red curves) and autofluorescence (blue curves) intensity profiles with respect to the distance $r$ from the excitation line.

Two types of simulations were performed to check the validity of our hypothesis: first with the Monte-Carlo method (with $10^{7}$ photons launched) and also with the NIRFAST software ${ }^{23,24}$ which is based on the diffusion approximation.

In both simulations, the medium is a homogeneous fluorescent one in a slab geometry with tissue-like optical properties $\left(\mu_{a}=0.05 \mathrm{~cm}^{-1}, \mu_{s}^{\prime}=10 \mathrm{~cm}^{-1}, g=0.9\right)$.

To experimentally validate our hypothesis, measurements were acquired on a homogeneous autofluorescent phantom. Fluorescence and excitation images were acquired. As with the simulations, we studied the profiles ratios $A / E$ for several increasing levels of autofluorescence.

The results obtained with both simulations and experimentally are presented on Fig. 4.

$A / E$ ratio profiles are presented for increasing levels of autofluorescence. We see the linear relationship of $A / E$ with $r$ as expected in all cases. For experimental data and a distance superior to $30 \mathrm{~mm}$, the noise due to lack of photons becomes dominant. Subsequent experimental analyzes will therfore only be done for distances to the source inferior to $20 \mathrm{~mm}$.

We will now explain how we take advantage of this relationship between the excitation and 

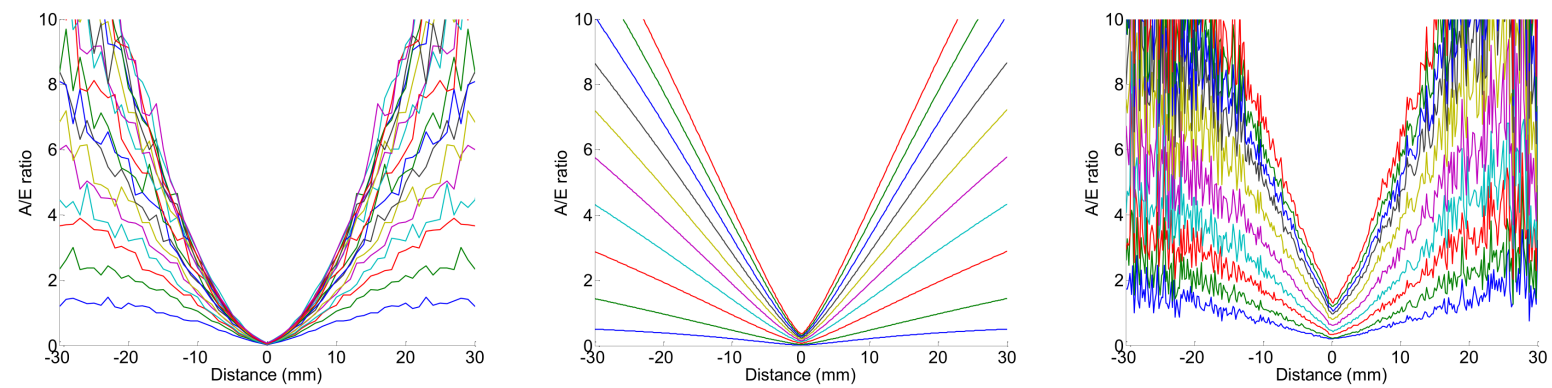

Fig $4 A$ / $E$ intensity ratios obtained for linearly increasing levels of autofluorescence with the Monte-Carlo method (left, 20 levels of autofluorescence considered), with NIRFAST (center, 10 levels of autofluorescence considered), and experimentally (right, 10 levels of autofluorescence considered).

autofluorescence signals to reduce the effects of autofluorescence.

Let us consider that, for one excitation position $i$ and one column of the images $j$ :

$$
\begin{gathered}
I_{t o t}(r, i, j) \approx F(r, i, j)+\alpha(i, j) \cdot E(r, i, j)+A(r, i, j) \\
I_{t o t}(r, i, j) \approx F(r, i, j)+\alpha(i, j) \cdot E(r, i, j)+\beta \cdot(i, j) \cdot E(r, i, j) . r
\end{gathered}
$$

where $I_{t o t}$ is the total signal detected on the camera, $F$ is the fluorescence signal of interest, $A$ is the autofluorescence, $E$ are excitation leaks, $\alpha$ and $\beta$ are coefficients depending on the excitation position and the column considered for the intensity profiles.

By fitting $I_{t o t}$ with $\alpha . E+\beta$.E.r, we can find the fluorescence signal of interest $F$ by subtraction.

The fitting method is the following: for each excitation position, we acquire both a fluorescence and an excitation image. For each position, we then fit the fluorescence intensity profile in each column of the image ( 480 columns with our detector) by using the corresponding excitation profile with the method of least squares. We then obtain $\alpha$ and $\beta$ parameters specific to each position. These parameters stay the same for excitation positions where only the autofluorescence is excited and have strong variations for positions surrounding the fluorescence target.

We studied two possibilities: we can either choose to use the parameters specific to each positions (referred as "local parameters") or to use the mean parameters (referred as "global parame- 
ters"). As we will see with the following simulations made with NIRFAST, each possibility has its own advantages and drawbacks.

In the first case studied, we used the same homogeneous fluorescent medium as in the previous simulation and we added a fluorescent inclusion located $2 \mathrm{~mm}$ below the surface. The simulated phantom and the different intensity profiles of interest are depicted on Fig. 5.

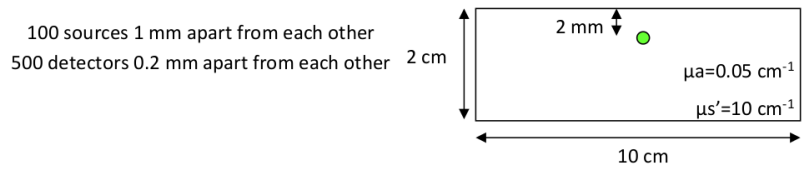

Fluorescent inclusion with 20 times the background fluorescence yield
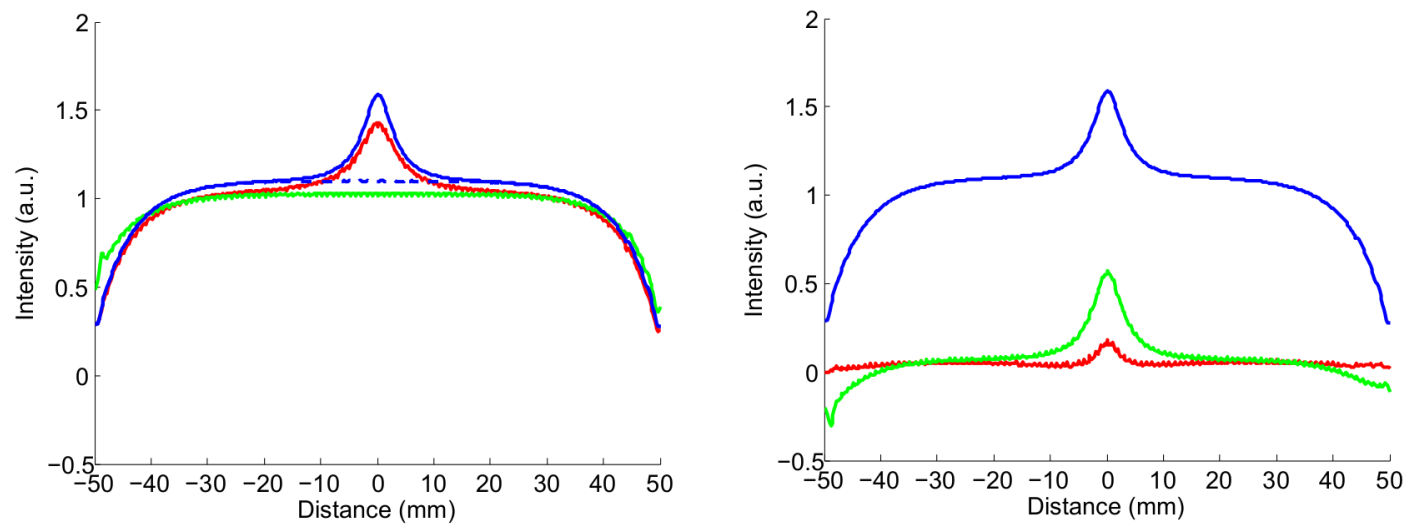

Fig 5 Intensity profiles comparison: left: - raw fluorescence $F$, - - - autofluorescence, - local parameters fit of the fluorescence $M_{1},-$ global parameters fit of the fluorescence $M_{2}$; right: $-F,-\left(F-M_{1}\right),-\left(F-M_{2}\right)$.

As expected, the intensity profile obtained when fitting with the local parameters (noted $M_{1}$, red curve on the left of Fig. 5) is close to the total fluorescence profile and is sensitive to the fluorescent inclusion, while the one obtained when using the global parameters (noted $M_{2}$, green curve on the left of Fig. 5) is closer to the autofluorescence profile and is not sensitive to the fluorescent inclusion.

After subtraction, both methods completely nullify the autofluorescence contribution. The global parameters method leads to a better dynamic around the fluorescence inclusion but suffers from boundary effects for distances larger than $40 \mathrm{~mm}$ from the inclusion in this case. 
For the second case studied, we used the same medium as in the previous simulation and we added five fluorescent inclusions located $2 \mathrm{~mm}$ below the surface. The simulated phantom and the different intensity profiles of interest are depicted on Fig. 6.
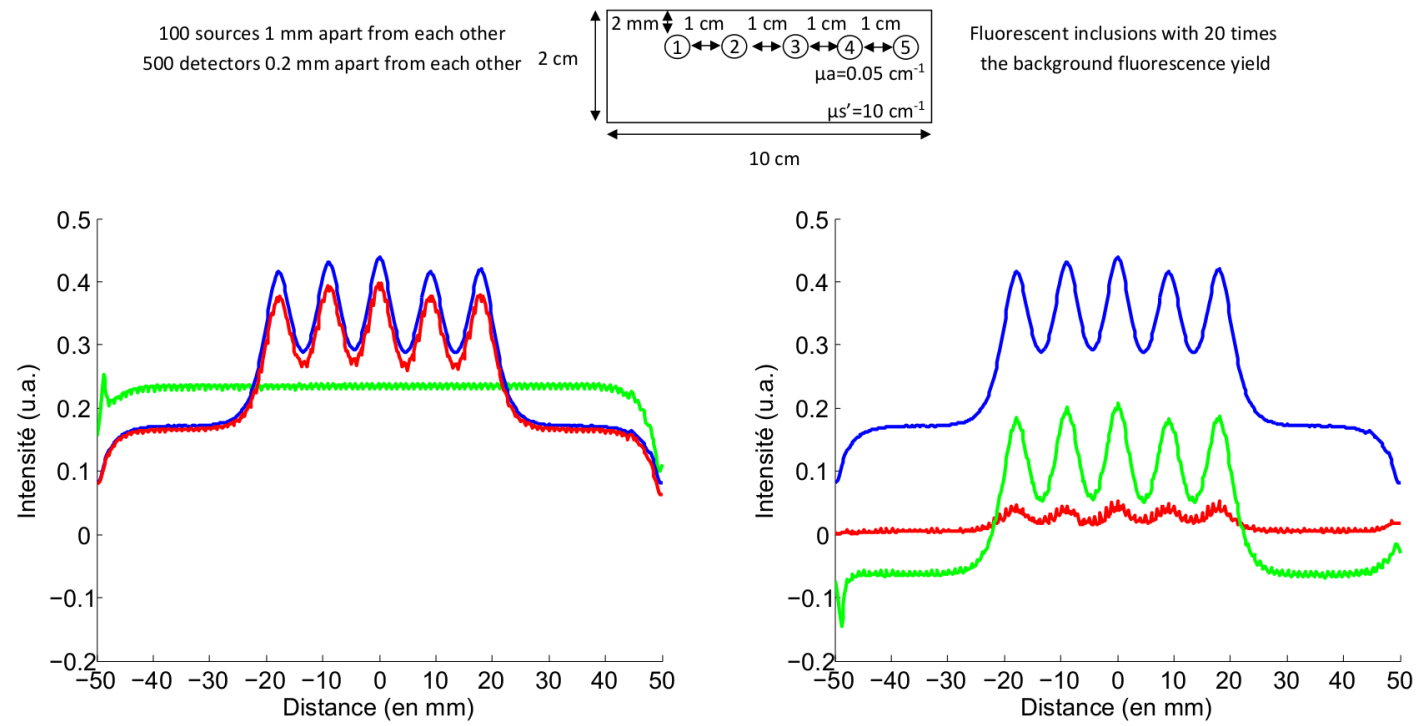

Fig 6 Intensity profiles comparison: left: - raw fluorescence $F$, - - - autofluorescence, - local parameters fit of the fluorescence $M_{1},-$ global parameters fit of the fluorescence $M_{2}$; right: $-F,-\left(F-M_{1}\right),-\left(F-M_{2}\right)$.

In the previous case, the parameters $\alpha$ and $\beta$ were only varying for the excitation positions surrounding the fluorescent inclusion. This is why taking their mean value allowed to get close to the value of excitation positions where the background signal was the only signal detected. In this case, the parameters $\alpha$ and $\beta$ also vary only for excitation positions surrounding the fluorescent inclusions. However, there are five inclusions in this case while the excited field has the same size. As the number of excitation positions having parameters which depend on the fluorescent inclusions is higher, taking their mean value leads to an overestimation of the autofluorescence. This is why after subtraction we obtain a negative level of signal with the global parameters.

Figure 7 presents how our model behaves experimentally for five different positions of excitation around a fluorescent inclusion. On the first line of this figure, we can see the fluorescence 


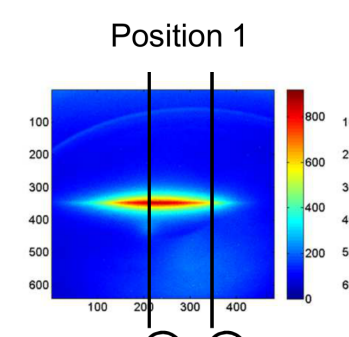

(1) (2)
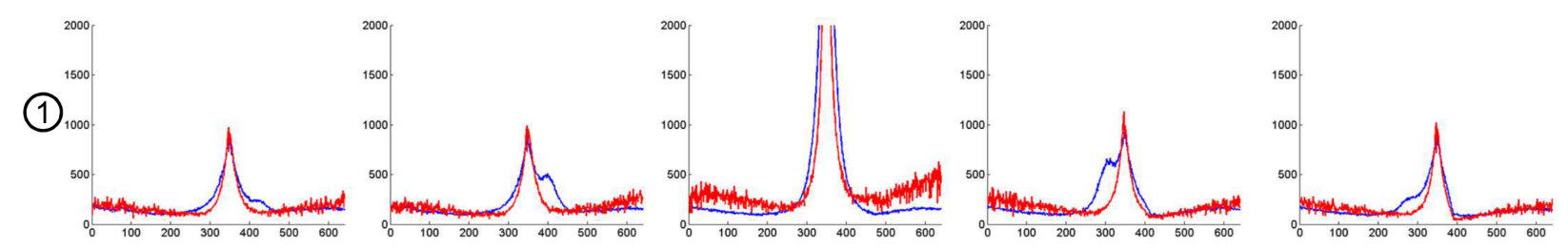

Fluorescence profile (-) and its fit ( - ) for column 1 at the 5 positions surrounding the inclusion
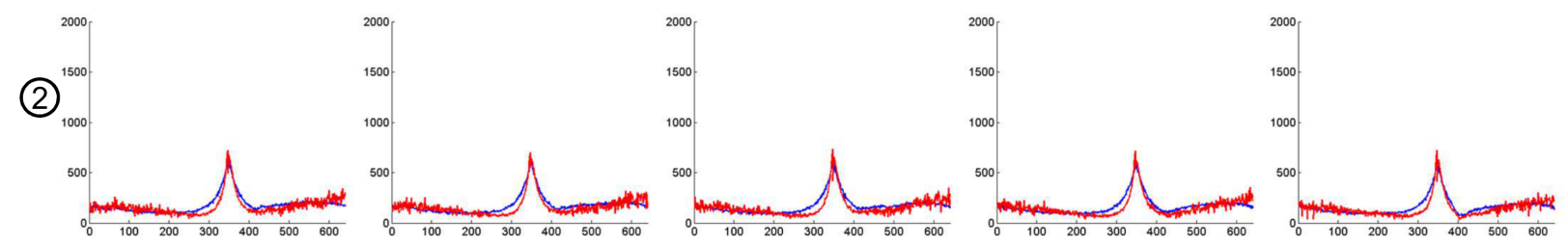

Fluorescence profile ( $(-)$ and its fit ( - ) for column 2 at the 5 positions where there is only autofluorescence

Fig 7 Example of fits obtained for five different positions of the excitation line around a fluorescent inclusion; top line: fluorescence images with the two columns used in this example, column 1 is above the fluorescent inclusion and column 2 is above autofluorescence only; center line: fluorescence intensity profile and its fit for column 1 for the five positions; bottom line: fluorescence intensity profile and its fit for column 2 for the five positions.

images and the two columns that will be used for the example: column 1 is located over the fluorescent inclusion and column 2 is located over an area where only the autofluorescence signal is detected. On the second line of the figure, the fluorescence intensity profiles and their fits are plotted for column 1 at the five positions considered. On the third line, the fluorescence intensity profiles and their fits are plotted for column 2 at the five positions considered.

On this last line where only the autofluorescence is considered, we see that the intensity profile and its fit overlap nearly perfectly. When subtracting its fit to the fluorescence intensity profile, the contribution from the autofluorescence is then completely suppressed.

On the other hand, on the second line where some of the fluorescence of interest is detected, some differences appear between the fluorescence intensity profile and its fit. These differences 
become more and more important as the excitation line is getting closer to the fluorescent inclusion. In this case, when the fit is subtracted to the fluorescence intensity profile, the autofluorescence contribution is suppressed but the fluorescence of interest contribution remains.

\section{Results and discussion}

\subsection{Contrast enhancement}

To compare the different detection schemes and quantify the improvements, we introduce the contrast $C_{T, N}$ defined as:

$$
C_{T, N}=\frac{\langle T\rangle-\langle N\rangle}{\langle T\rangle+\langle N\rangle}
$$

where $\langle T\rangle$ and $\langle N\rangle$ are respectively the mean intensity values in a target region of interest (with fluorescence) and in a neutral region of interest (with background fluorescence only).

The regions of interest were the same for both WF-FRI and our method. The neutral region of interest was chosen in the top edge of the images so that it was the farthest possible from the target region of interest while still being illuminated the same way, to ensure that the inhomogeneities of the illumination could not bias the results.

We will first present how our method improves the contrast compared to the classical WF-FRI in the case of a phantom with a single fluorescent inclusion at different increasing depths. Two different levels of background fluorescence were considered: a realistic one, and a stronger one to test the limits of the method. The realistic level of background fluorescence has been chosen using Ref. 25. We have used a concentrations ratio of about 80 between the fluorescent inclusion and the background medium (this is based on the "ICG equivalent" signal of skin which is the median 
one compared to the different organs presented in Ref. 25). For the stronger level of background fluorescence, we used a ratio of fluorescence to background fluorescence five times lower. We then had an "ICG equivalent" autofluorescence signal 1.5 times higher than the worst case described in Ref. 25. This extreme case allows us to test the limits of our method and could be compared to a case right after the fluorescence dye is injected in the patient, leading to a strong homogeneous non specific fluorescence signal which would be added to the existing background fluorescence signal.

On the left of Fig. 8, we compare the contrasts observed at the ten depths considered with the WF-FRI and both fitting methods for the realistic level of background fluorescence.
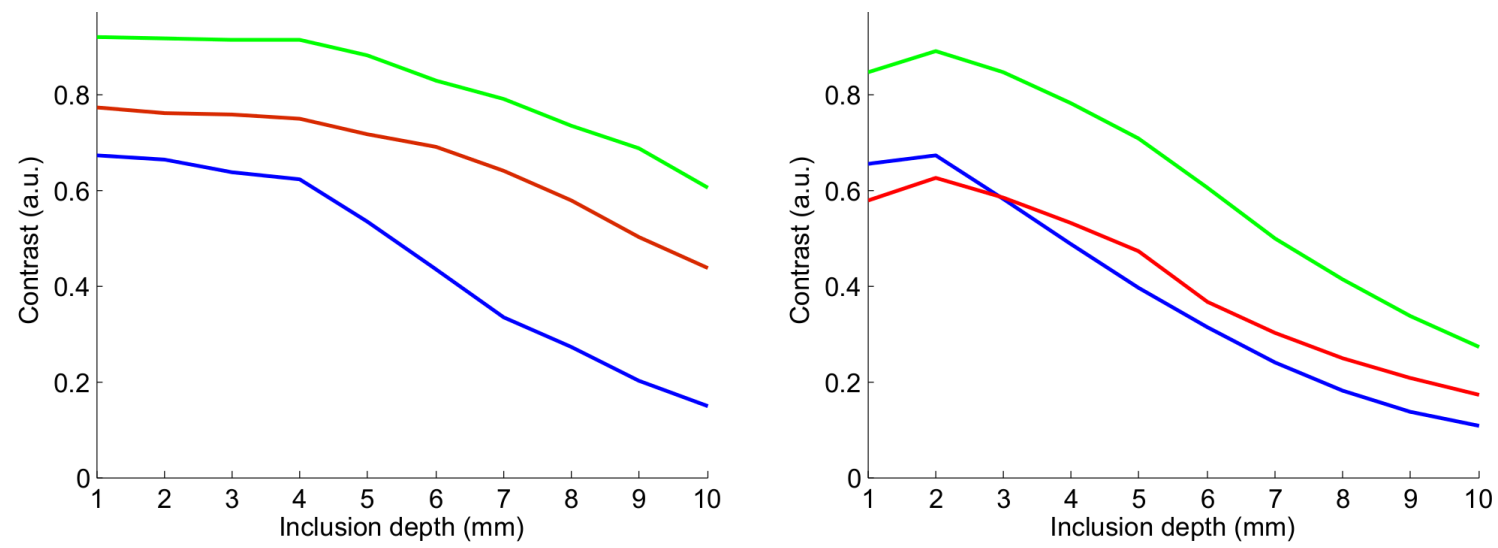

Fig 8 Comparison between the contrasts obtained with WF-FRI (-), the local parameters fitting method (-) and the global parameters fitting method (-) for a realistic background fluorescence level (left) and a stronger background fluorescence level (right) used to test the limits of the method.

Contrast is enhanced with both the local and global parameters fitting methods. In this simple case where there is a single fluorescent inclusion, the global parameters method offers better performance as mentioned before: with the global parameters method, the parameters used are the means on every excitation position. By doing this, the "fast" fluorescence variations corresponding to fluorescence inclusions or absorption heterogeneities are lost, but we are closer to the "slow" fluorescence variations corresponding to autofluorescence. This is why after subtracting the fit to 
the fluorescence image we obtain a very good contrast as we subtract only the autofluorescence contribution. In this case, the gain obtained with the local parameters varies between 1.2 at $1 \mathrm{~mm}$ and 3.1 at $10 \mathrm{~mm}$ while it varies between 1.4 and 4.4 with the global parameters.

On the right of Fig. 8, we present the results in the same case as before but with a stronger background fluorescence signal.

Even in the case with a stronger background signal, our fitting method enhances the contrast compared to the WF-FRI with both local and global parameters. However, the gain obtained is smaller than the one obtained in the case with less background signal. With the local parameters fitting method, the gain is low, with a maximum of 1.5 at $10 \mathrm{~mm}$. With the global parameters fitting method, the gain varies between 1.3 at $1 \mathrm{~mm}$ and 2.3 at $10 \mathrm{~mm}$.

\subsection{Resolution enhancement}

The second set of results was obtained with the fluorescent resolution target described in section 2.1. The concentration of background fluorescence was set to have a realistic fluorescence to background ratio (as described before), and the target was immerged at three depths between 1 $\mathrm{mm}$ and $3 \mathrm{~mm}$. The images obtained are presented on Fig. 9.

At $1 \mathrm{~mm}$, it is possible to resolve the target with WF-FRI (left column) but there is already some crosstalk between the different groups of inclusions leading to an overestimation of the signal produced by the largest inclusions.

The local parameters fitting method (central column) offers the best performance. It enhances the resolution as it increases the peak to valley ratio between the fluorescent inclusions and the background, and the background fluorescence surrounding the inclusions is totally suppressed. The global parameters fitting method (right column) also suppresses the background fluorescence 


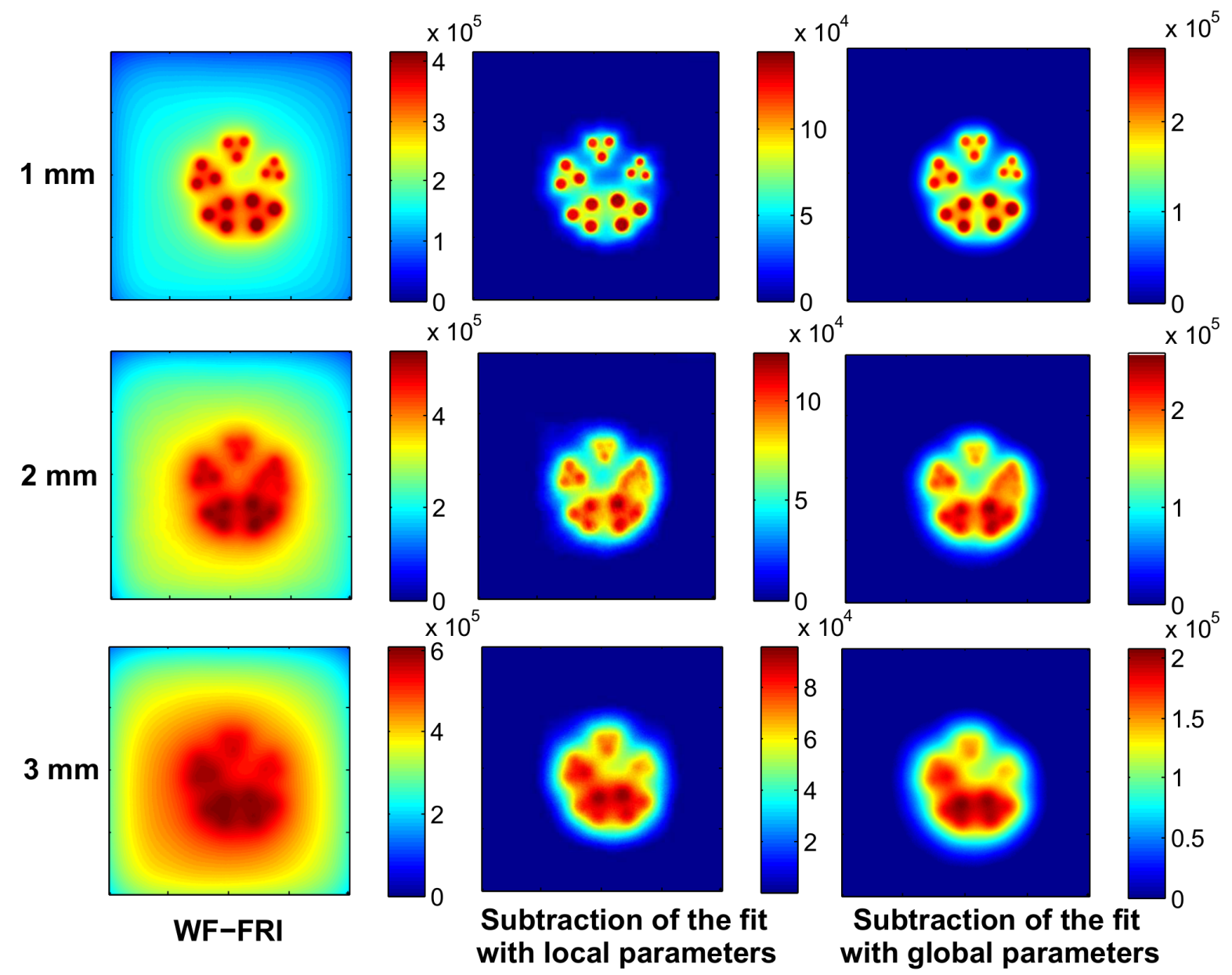

Fig 9 Images of the resolution target at three different depths obtained with WF-FRI (left column), the local parametes fitting method (central column) and the global parameters fitting method (right column).

surrounding the inclusions, but the peak to valley ratio between the fluorescent inclusions and the background is slightly lower than with the local parameters fitting method.

At $2 \mathrm{~mm}$, it becomes difficult to properly resolve the target with WF-FRI. Signals coming from the three largest groups of inclusions start to overlap and form one large fluorescent signal, making it impossible to distinguish the single inclusions in some groups.

The local parameters fitting method still offers the best performance as the resolution between the groups of inclusions is still good, but it becomes harder to see the inclusions in the group of inclusions with the smallest diameter. The global parameters fitting method gives results compa- 
rable to the ones obtained with the local parameters fitting method, but, as seen at $1 \mathrm{~mm}$, the peak to valley ratio between the fluorescent inclusions and the background is slightly lower.

At $3 \mathrm{~mm}$, the whole target only emits one large fluorescent signal with WF-FRI and the groups of inclusions are completely unresolved. With both fitting methods, the target is unresolved and we can only see one large signal due to the three largest groups of inclusions and two smallest signals due to the two smallest groups of inclusions.

To conclude on these results, the local parameters fitting method is better suited to enhance the resolution then the global parameters fitting method. As mentioned before, with the local parameters, the parameters used are the ones specific to each position. Contrary to the mean parameters, we do not lose all the "fast" fluorescence variations, this is why the subtraction of the fit leads to a better resolution and a correction of some effects of the absorption heterogeneities.

However, the main interest of these fitting method is the reduction of the background fluorescence signal.

\subsection{Effect of the absorption}

We wanted to test the robustness of our method in conditions where absorption heterogeneities are present. Two simple yet interesting cases were studied: a first one where an absorption heterogeneity is located right above a fluorescent inclusion, giving the impression that two fluorescent inclusions are present rather than one, and a second one where two fluorescent inclusions with the same fluorescence but different absorptions give different signals. The aim is not to fully correct the absorption effects but rather to test the limits of our method. In this section, we compare the results obtained with our fitting methods to the WF-FRI as previously, but also to the fluorescence by excitation ratio method presented in Ref. ${ }^{26}$ 
The first phantom used for this study is the one described in Fig. 2.b. A fluorescent inclusion is located $2 \mathrm{~mm}$ below the surface of the phantom and an absorption inclusion is right above it. Four different absorption coefficients were used for the absorption inclusion: $0.05 \mathrm{~mm}^{-1}$ (matching with the surrounding absorption of the phantom), $0.1 \mathrm{~mm}^{-1}, 0.2 \mathrm{~mm}^{-1}, 0.5 \mathrm{~mm}^{-1}$. On Fig. 10, we show the images obtained with the WF-FRI, both fitting methods, and the fluorescence by excitation ratio method for the control experiment without heterogeneity and for the experiment with the highest absorption coefficient considered.

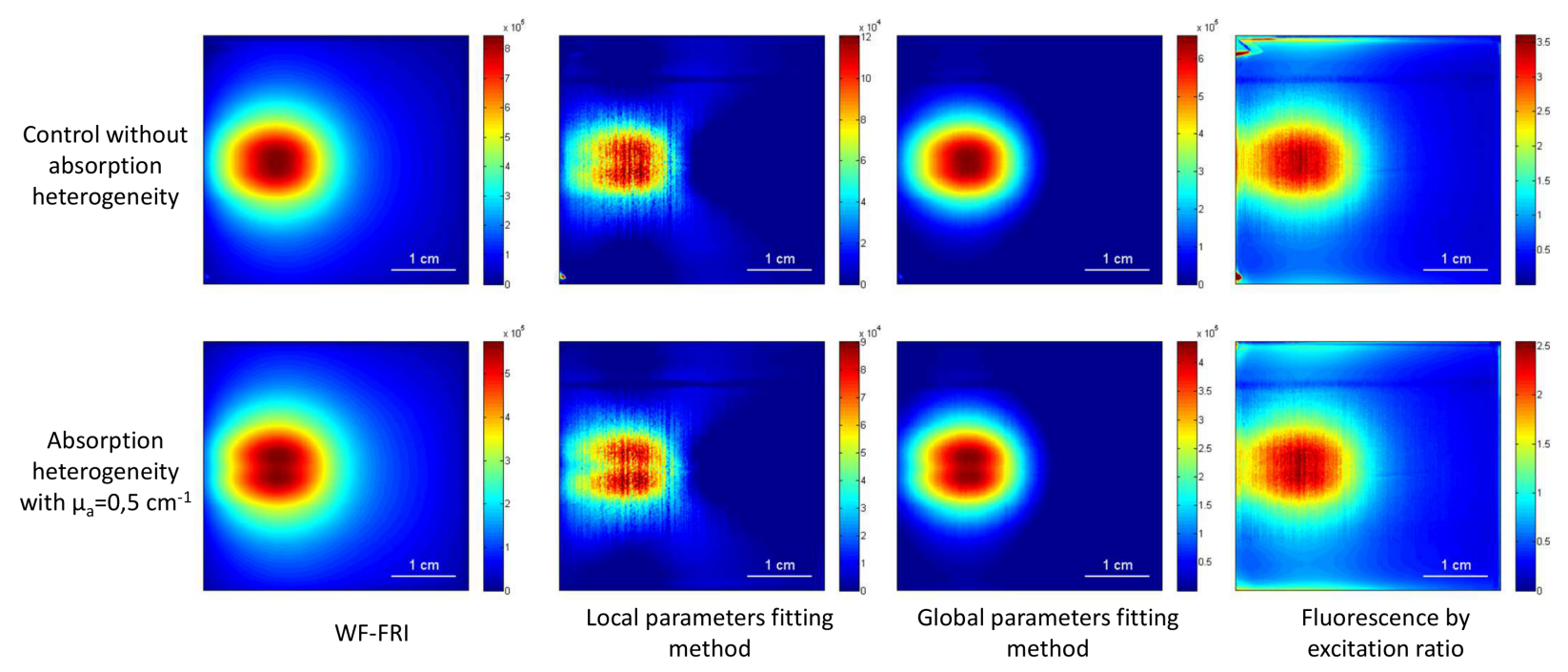

Fig 10 Images obtained with the different methods for two configurations: top line: control experiment, the heterogeneity has the same absorption coefficient as the surrounding phantom; bottom line: the heterogeneity has an absorption coefficient ten times higher than the surrounding phantom.

We see on the WF-FRI images the influence of the absorption heterogeneity: due to the decrease of the fluorescence signal caused by the heterogeneity, it seems that there are two fluorescence sources rather than a single one. The same behaviour is observed for both fitting methods. However, the fluorescence by excitation ratio method corrects this effect as the signal detected is the same for the control experiment and the heterogeneity experiment.

These remarks are best visualized on Fig. 11 where we have plotted, the intensity profiles 
taken perpendicularly to the capillary for the different methods. The profiles for the four different absorption coefficients are shown.

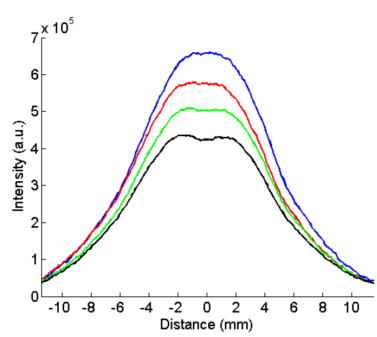

WF-FRI

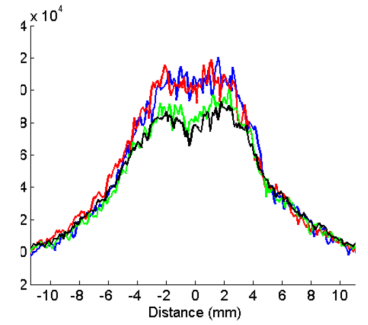

Local parameters fitting method

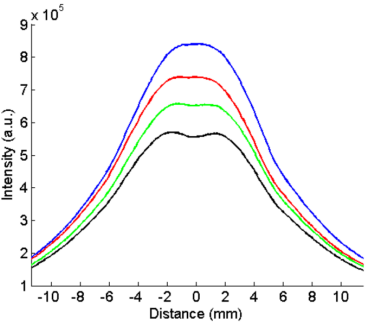

Global parameters fitting method

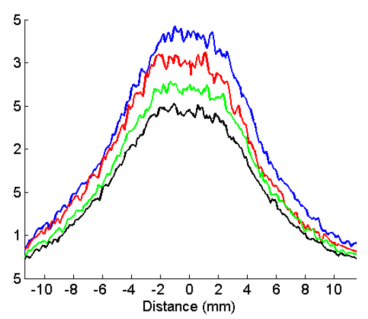

Fluorescence by excitation ratio

Fig 11 Fluorescence intensity profiles obtained with the different methods for four absorption coefficient for the heterogeneity: $0.05 \mathrm{~mm}^{-1}(-), 0.1 \mathrm{~mm}^{-1}(-), 0.2 \mathrm{~mm}^{-1}(-), 0.5 \mathrm{~mm}^{-1}(-)$.

With WF-FRI, we see that the drop of intensity becomes more and more visible as the absorption coefficient of the heterogeneity increases. As said previously, for the highest absorption considered, two different peaks begin to appear.

With the global parameters fitting method, no improvement is observed, the behaviour is comparable to the one observed with WF-FRI.

With the local parameters fitting method, we see that the two peaks caused by the heterogeneity are still visible and are not corrected. However, we see that the intensity decrease due to the absorption is partly corrected: with WF-FRI, the signal decreases at $83 \%$ of the control experiment with the $\mu_{a}=0.1 \mathrm{~mm}^{-1}$ heterogeneity, $71 \%$ with the $\mu_{a}=0.2 \mathrm{~mm}^{-1}$ heterogeneity and $57 \%$ with the $\mu_{a}=0.5 \mathrm{~mm}^{-1}$ heterogeneity. With the local parameters fitting method, the signal stays at $100 \%$ of the control experiment with the $\mu_{a}=0.1 \mathrm{~mm}^{-1}$ heterogeneity, decreases at $75 \%$ with the $\mu_{a}=0.2 \mathrm{~mm}^{-1}$ heterogeneity and $75 \%$ with the $\mu_{a}=0.5 \mathrm{~mm}^{-1}$ heterogeneity.

With the fluorescence by excitation ratio method, we see that the two peaks caused by the heterogeneity are corrected. We also see that the intensity decrease due to the absorption is partly 
corrected: the signal decreases at $88 \%$ of the control experiment with the $\mu_{a}=0.1 \mathrm{~mm}^{-1}$ heterogeneity, $75 \%$ with the $\mu_{a}=0.2 \mathrm{~mm}^{-1}$ heterogeneity and $60 \%$ with the $\mu_{a}=0.5 \mathrm{~mm}^{-1}$ heterogeneity.

To conclude on these first results, the local parameters fitting method seems to be suited to correct some of the effects of absorption heterogeneities, namely the decrease of the intensity detected. The results are slightly better than the fluorescence by excitation ratio, but this method also manages to correct the shape changes of the signal detected which are caused by the heterogeneities : there is only one flat peak compared to the local parameters fitting method which gives two peaks.

The second phantom used for this study is the one described in Fig. 2.c. Two fluorescent capillaries are located at the same depth, the distance between them is $1 \mathrm{~cm}$. Two experiments were performed: one where both fluorescent inclusions have the same absorption coefficient as the background, and another one where one of the inclusions has an absorption coefficient ten times higher (the top capillary on Fig. 12). The images obtained with the WF-FRI, both fitting methods, and the fluorescence by excitation ratio method are shown on Fig. 12.

As expected, the higher absorption of the top capillary leads to a decrease of its signal intensity : it is twice as low as the one of the capillary having the same absorption as the background. None of the techniques seem to be able to correct the effects of absorption.

To have a quantitative idea of the absorption correction of the different techniques, we have plotted on Fig. 13 the intensity profiles taken perpendicularly to the capillaries for the different methods. 


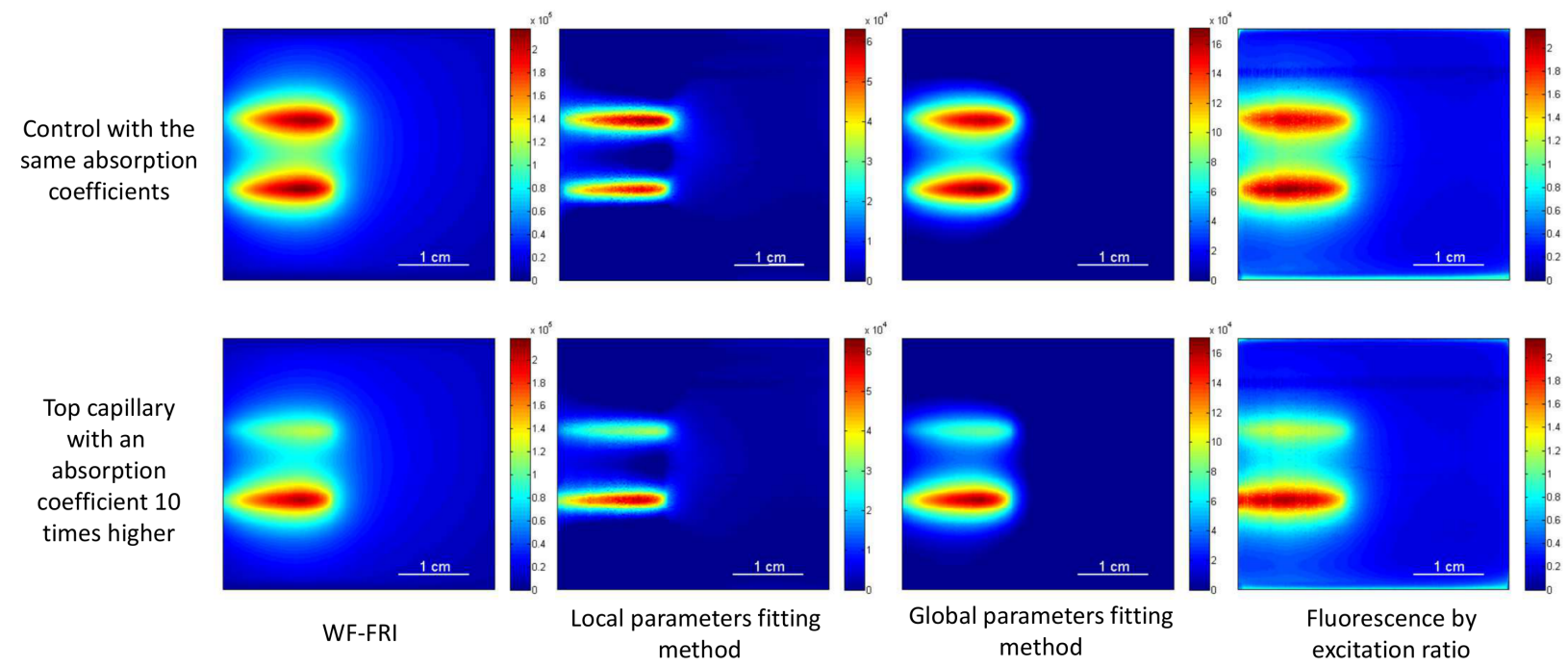

Fig 12 Images obtained with the different methods for two configurations: top line: both capillaries have the same absorption coefficient; bottom line: the top capillary has an absorption coefficient ten times higher.

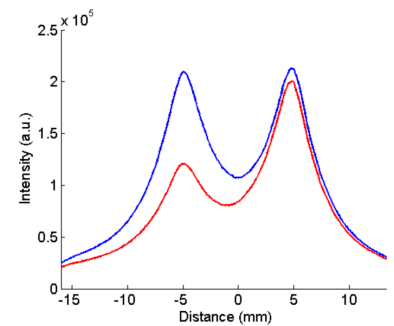

WF-FRI

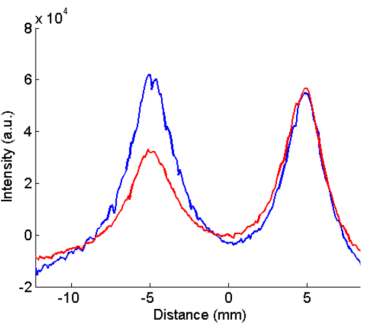

Local parameters fitting method

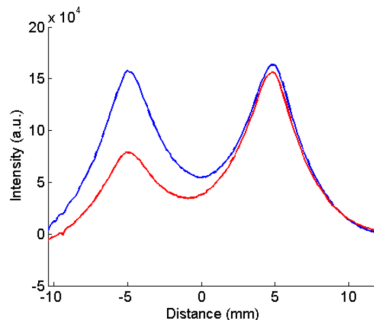

Global parameters fitting method

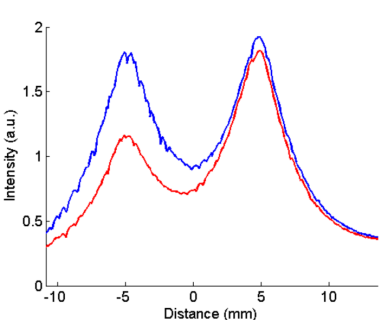

Fluorescence by excitation ratio

Fig 13 Fluorescence intensity profiles obtained with the different methods for two configurations: both capillaries have the same absorption coefficient (—), the left capillary has an absorption coefficient ten times higher(-).

First, we can notice that the local parameters fitting method enhances the resolution compared to other methods, as it was shown in the previous section. It also appears that no method totally corrects the effects of absorption. The only method that increases the signal of the higher absorption capillary is the local parameters fitting method: the signal is at $63 \%$ of its original level, contrary to the other methods where it is at $50 \%$.

As in the previous case, the local parameters fitting method is suited to correct some of the effects of absorption heterogeneities, even in this case where the fluorescence by excitation ratio has no beneficial effect. 


\section{Conclusion}

We presented in this study a novel approach for molecular imaging based on the use of a laser line illumination rather than the more classical WF-FRI. By using a laser line to illuminate the object to study and acquiring fluorescence and excitation images for each position of the line, we can use the relationship existing between the excitation and autofluorescence intensity profiles to enhance the contrast and resolution of the signals. The technique has been tested on different phantoms mimicking tissue-like optical properties.

While a strong background fluorescence can reduce the performance of the method, we can still expect a noticeable improvement compared to the WF-FRI. We also showed that this technique can be an alternative to the fluorescence by excitation ratio method used by some groups to account for the absorption heterogeneities. We saw that in the simple cases considered the technique does not give aberrant results and can partly correct these detrimental effects better than the ratio method.

All the results presented here have been obtained by post-processing the images to do a proof of principle. However, we are currently working on the optical setup to be able to apply it in realtime, permitting its use in a real clinical application. The main challenge will be the simultaneous acquisition of fluorescence and excitation and the implementation of the image processing at a speed suitable for real-time imaging.

\section{References}

1 J. V. Frangioni, "In vivo near-infrared fluorescence imaging," Current opinion in chemical biology 7, pp. 626-634, Oct. 2003. PMID: 14580568.

2 V. Ntziachristos, C. Bremer, and R. Weissleder, "Fluorescence imaging with near-infrared 
light: new technological advances that enable in vivo molecular imaging," European radiology 13, pp. 195-208, Jan. 2003. PMID: 12541130.

3 E. M. Sevick-Muraca, J. P. Houston, and M. Gurfinkel, "Fluorescence-enhanced, near infrared diagnostic imaging with contrast agents," Current opinion in chemical biology $\mathbf{6}$, pp. 642-650, Oct. 2002. PMID: 12413549.

4 S. A. Hilderbrand and R. Weissleder, "Near-infrared fluorescence: application to in vivo molecular imaging," Current opinion in chemical biology 14, pp. 71-79, Feb. 2010. PMID: 19879798.

5 J. Hung, S. Lam, J. C. Leriche, and B. Palcic, "Autofluorescence of normal and malignant bronchial tissue," Lasers in Surgery and Medicine 11(2), pp. 99-105, 1991.

6 S. Gioux, H. S. Choi, and J. V. Frangioni, "Image-guided surgery using invisible near-infrared light: fundamentals of clinical translation," Molecular imaging 9, pp. 237-255, Oct. 2010.

7 S. Bhaumik, J. DePuy, and J. Klimash, "Strategies to minimize background autofluorescence in live mice during noninvasive fluorescence optical imaging," Lab Animal 36, pp. 40-43, Sept. 2007.

8 Y. Inoue, K. Izawa, S. Kiryu, A. Tojo, and K. Ohtomo, "Diet and abdominal autofluorescence detected by in vivo fluorescence imaging of living mice," Molecular Imaging 7, pp. 21-27, Feb. 2008.

9 S. MacLaurin, M. Bouchard, P. Dwyer, L. R., J. Mansfield, and T. Krucker, "Reduction of skin and food autofluorescence in different mouse strains through diet changes," tech. rep., 2006. 
10 D. Wood, G. Feke, D. Vizard, and R. Papineni, "Refining epifluorescence imaging and analysis with automated multiple-band flat-field correction," Nature Methods 5, Apr. 2008.

11 J. R. Mansfield, K. W. Gossage, C. C. Hoyt, and R. M. Levenson, "Autofluorescence removal, multiplexing, and automated analysis methods for in-vivo fluorescence imaging," Journal of biomedical optics 10, p. 41207, Aug. 2005.

12 H. Xu, C. Kuo, and B. Rice, "Improved sensitivity by applying spectral unmixing prior to fluorescent tomography," in Biomedical Optics, OSA Technical Digest (CD), p. BMC1, Optical Society of America, Mar. 2008.

13 C. H. V. d. Lest, E. M. Versteeg, J. H. Veerkamp, and T. H. V. Kuppevelt, "Elimination of autofluorescence in immunofluorescence microscopy with digital image processing.," Journal of Histochemistry \& Cytochemistry 43, pp. 727-730, July 1995.

14 T. Troy and B. Rice, "Methods for reducing the effects of background autofluorescence using IVIS imaging technology,” tech. rep., 2004.

15 W. Wang, S. Demos, J. Ali, G. Zhang, and R. Alfano, "Visibility enhancement of fluorescent objects hidden in animal tissues using spectral fluorescence difference method," Optics Communications 147, pp. 11-15, Feb. 1998.

16 M. Neumann and D. Gabel, "Simple method for reduction of autofluorescence in fluorescence microscopy," Journal of Histochemistry \& Cytochemistry 50, pp. 437-439, Mar. 2002.

17 W. Pham, L. Cassell, A. Gillman, D. Koktysh, and J. C. Gore, "A near-infrared dye for multichannel imaging," Chemical communications (Cambridge, England), pp. 1895-1897, Apr. 2008.

18 R. Kumar, T. Y. Ohulchanskyy, I. Roy, S. K. Gupta, C. Borek, M. E. Thompson, and P. N. 
Prasad, "Near-infrared phosphorescent polymeric nanomicelles: efficient optical probes for tumor imaging and detection," ACS applied materials \& interfaces 1, pp. 1474-1481, July 2009.

19 W. Akers, F. Lesage, D. Holten, and S. Achilefu, "In vivo resolution of multiexponential decays of multiple near-infrared molecular probes by fluorescence lifetime-gated whole-body time-resolved diffuse optical imaging," Molecular imaging 6, pp. 237-246, Aug. 2007.

20 F. Fantoni, L. Hervé, V. Poher, S. Gioux, J. I. Mars, and J.-M. Dinten, "Laser line scanning for fluorescence reflectance imaging: a phantom study and in vivo validation of the enhancement of contrast and resolution,” Journal of Biomedical Optics 19, p. 106003, 2014.

21 F. P. Navarro, M. Berger, M. Goutayer, S. Guillermet, V. Josserand, P. Rizo, F. Vinet, and I. Texier, "A novel indocyanine green nanoparticle probe for non invasive fluorescence imaging in vivo," pp. 71900L-71900L-10, SPIE, 2009.

22 A. Soubret and V. Ntziachristos, "Fluorescence molecular tomography in the presence of background fluorescence," Physics in medicine and biology 51, pp. 3983-4001, Aug. 2006. PMID: 16885619 .

23 H. Dehghani, M. E. Eames, P. K. Yalavarthy, S. C. Davis, S. Srinivasan, C. M. Carpenter, B. W. Pogue, and K. D. Paulsen, "Near infrared optical tomography using NIRFAST: algorithm for numerical model and image reconstruction," Communications in numerical methods in engineering 25, pp. 711-732, Aug. 2008. PMID: 20182646.

24 M. Jermyn, H. Ghadyani, M. A. Mastanduno, W. Turner, S. C. Davis, H. Dehghani, and B. W. Pogue, "Fast segmentation and high-quality three-dimensional volume mesh creation 
from medical images for diffuse optical tomography," Journal of Biomedical Optics 18(8), pp. 086007-086007, 2013.

25 A. M. De Grand, S. J. Lomnes, D. S. Lee, M. Pietrzykowski, S. Ohnishi, T. G. Morgan, A. Gogbashian, R. G. Laurence, and J. V. Frangioni, “Tissue-like phantoms for near-infrared fluorescence imaging system assessment and the training of surgeons," Journal of biomedical optics 11(1), p. 014007, 2006.

26 G. Themelis, J. S. Yoo, K.-S. Soh, R. Schulz, and V. Ntziachristos, "Real-time intraoperative fluorescence imaging system using light-absorption correction," Journal of biomedical optics 14, p. 064012, Dec. 2009.

\section{List of Figures}

1 Optical setup used during the study (schematic (a) and platform (b))

2 (a): Fluorescence target used for the resolution study; (b),(c): Phantoms used for the absorption correction study; (d): LNP-ICG fluorescence excitation and emission spectra.

3 Schematic of the geometry studied: a homogeneous fluorescent medium is considered and we look at the excitation (red curves) and autofluorescence (blue curves) intensity profiles with respect to the distance $r$ from the excitation line.

$4 A / E$ intensity ratios obtained for linearly increasing levels of autofluorescence with the Monte-Carlo method (left, 20 levels of autofluorescence considered), with NIRFAST (center, 10 levels of autofluorescence considered), and experimentally (right, 10 levels of autofluorescence considered). 
5 Intensity profiles comparison: left: - raw fluorescence $F$, - - - autofluorescence, — local parameters fit of the fluorescence $M_{1}$, - global parameters fit of the fluorescence $M_{2}$; right: $-F,-\left(F-M_{1}\right),-\left(F-M_{2}\right)$.

6 Intensity profiles comparison: left: - raw fluorescence $F$, - - - autofluorescence, — local parameters fit of the fluorescence $M_{1}$, — global parameters fit of the fluorescence $M_{2}$; right: $-F,-\left(F-M_{1}\right),-\left(F-M_{2}\right)$.

7 Example of fits obtained for five different positions of the excitation line around a fluorescent inclusion; top line: fluorescence images with the two columns used in this example, column 1 is above the fluorescent inclusion and column 2 is above autofluorescence only; center line: fluorescence intensity profile and its fit for column 1 for the five positions; bottom line: fluorescence intensity profile and its fit for column 2 for the five positions.

8 Comparison between the contrasts obtained with WF-FRI (-), the local parameters fitting method (-) and the global parameters fitting method (-) for a realistic background fluorescence level (left) and a stronger background fluorescence level (right) used to test the limits of the method.

9 Images of the resolution target at three different depths obtained with WF-FRI (left column), the local parametes fitting method (central column) and the global parameters fitting method (right column).

10 Images obtained with the different methods for two configurations: top line: control experiment, the heterogeneity has the same absorption coefficient as the surrounding phantom; bottom line: the heterogeneity has an absorption coefficient ten times higher than the surrounding phantom. 
11 Fluorescence intensity profiles obtained with the different methods for four absorption coefficient for the heterogeneity: $0.05 \mathrm{~mm}^{-1}(-), 0.1 \mathrm{~mm}^{-1}(-), 0.2 \mathrm{~mm}^{-1}$ $(-), 0.5 \mathrm{~mm}^{-1}(-)$.

12 Images obtained with the different methods for two configurations: top line: both capillaries have the same absorption coefficient; bottom line: the top capillary has an absorption coefficient ten times higher.

13 Fluorescence intensity profiles obtained with the different methods for two configurations: both capillaries have the same absorption coefficient (-), the left capillary has an absorption coefficient ten times higher(-). 


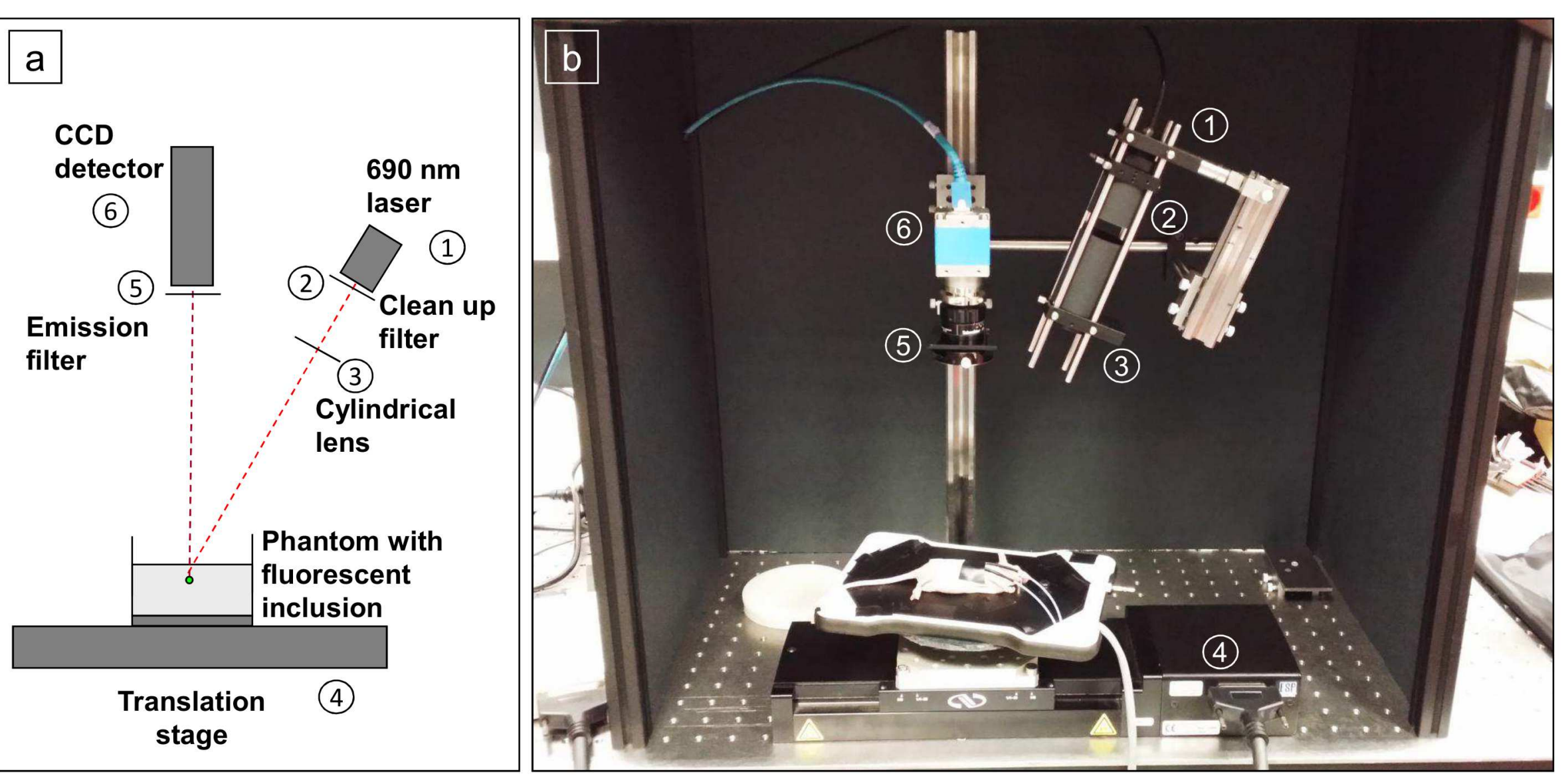




\section{a}

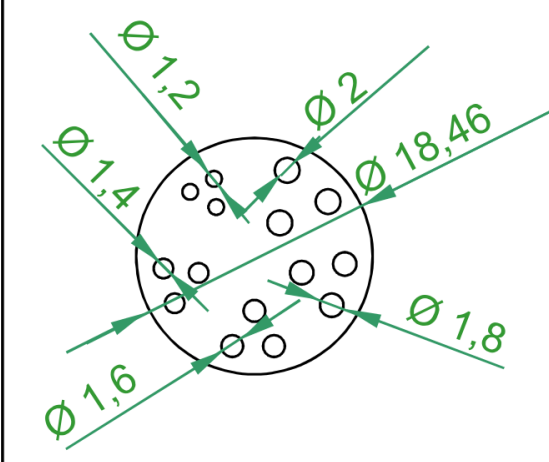

(dimensions in $\mathrm{mm}$ )
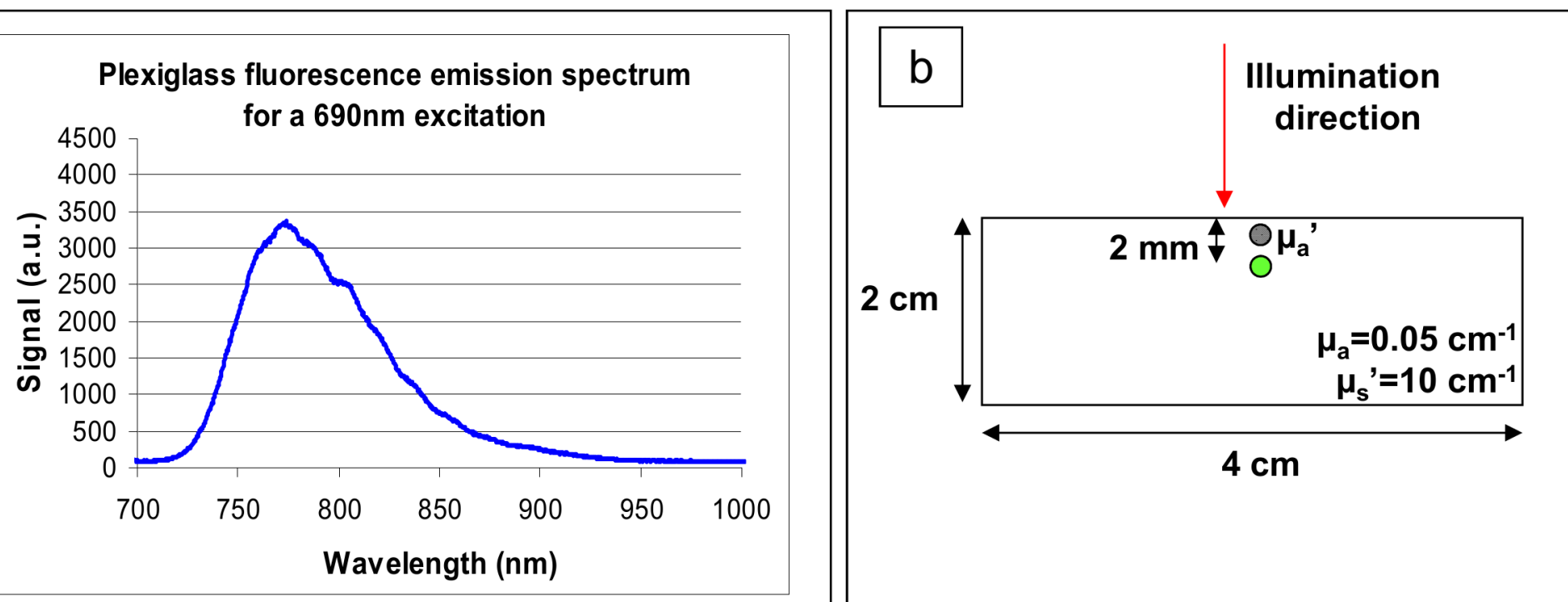

C
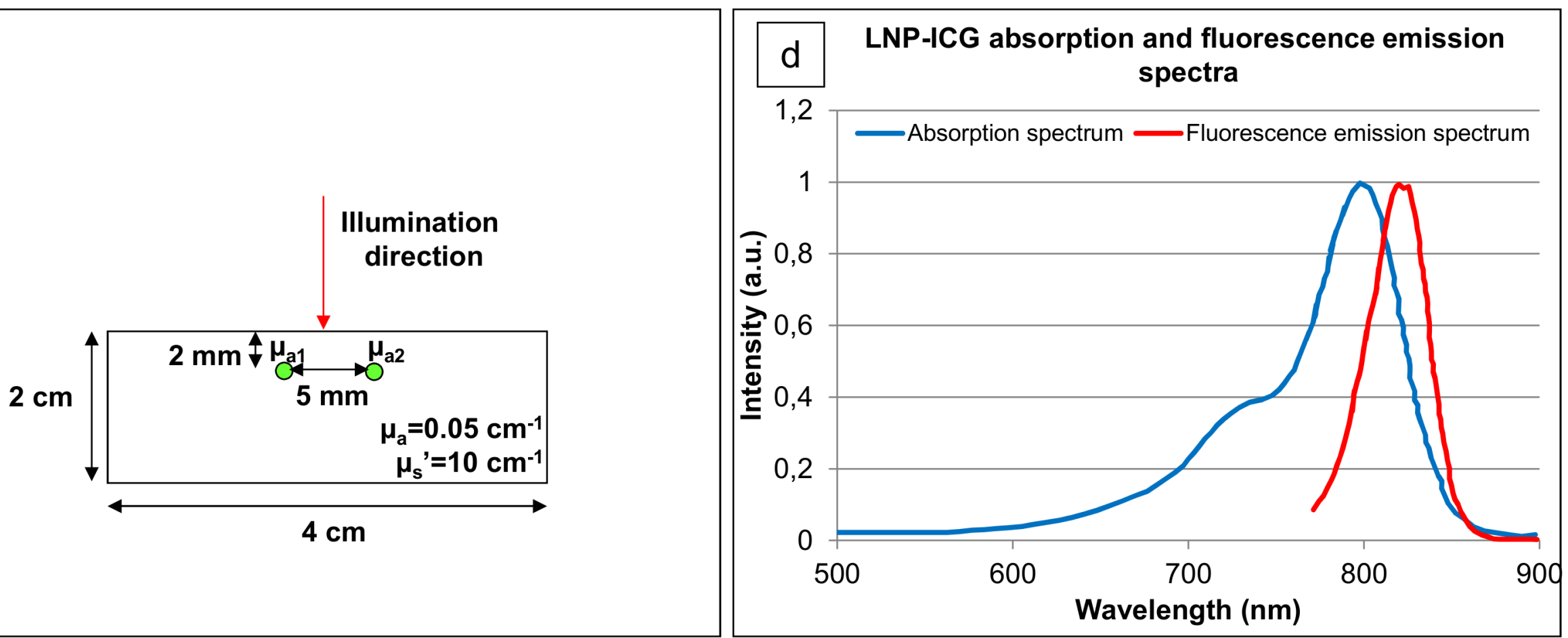


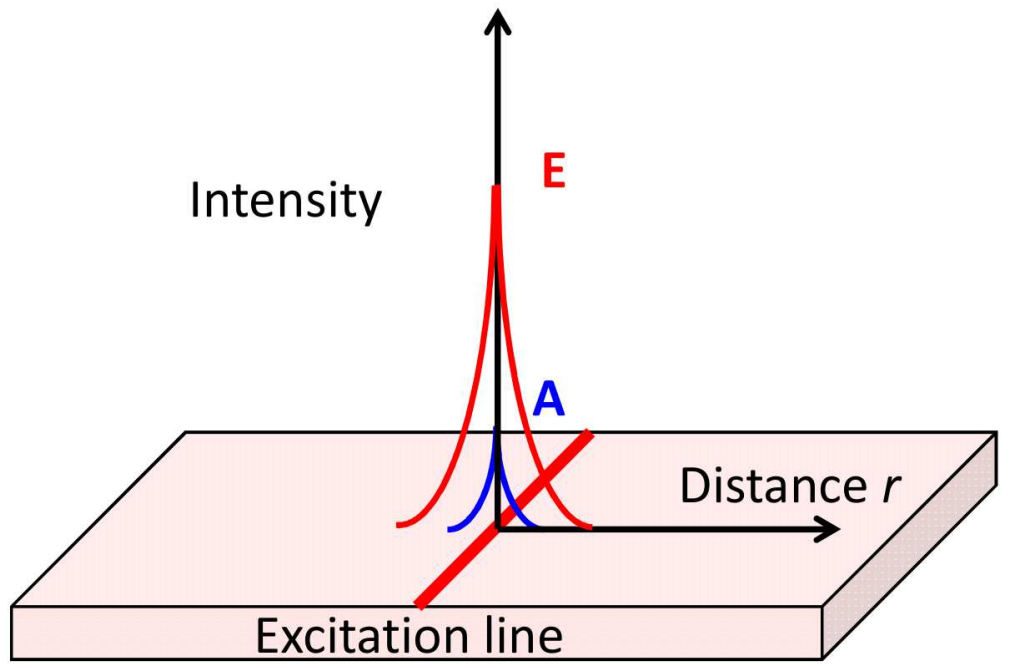

Homogeneous fluorescent medium 


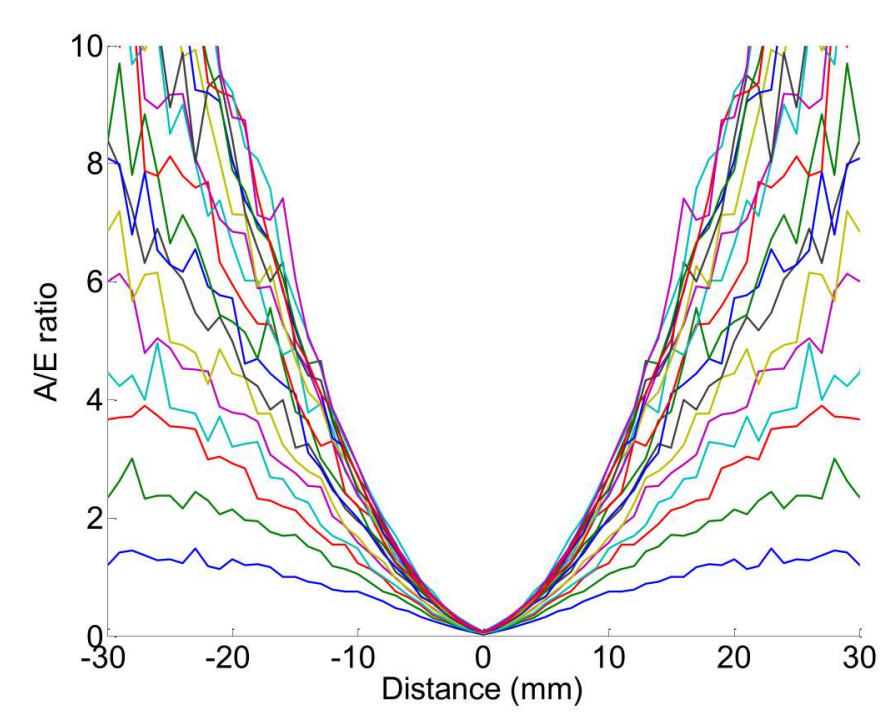



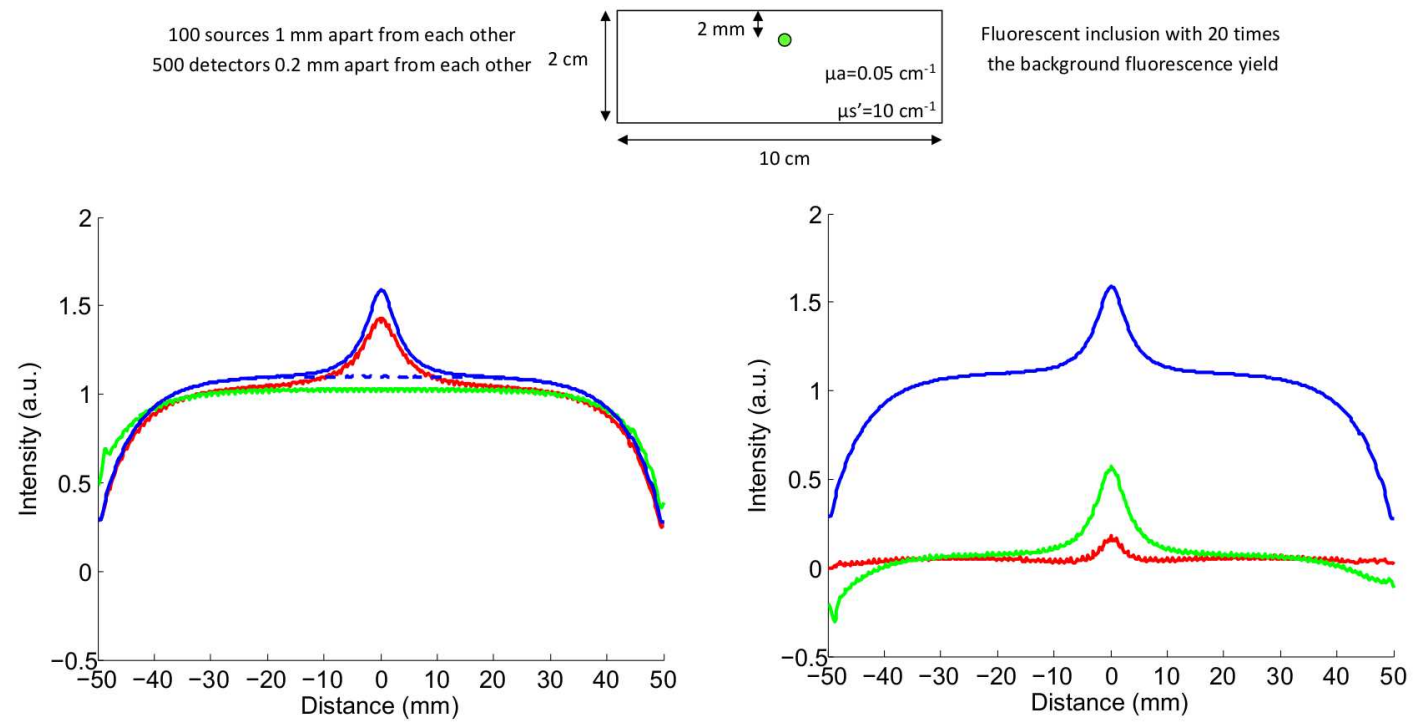
100 sources $1 \mathrm{~mm}$ apart from each other 500 detectors $0.2 \mathrm{~mm}$ apart from each other $2 \mathrm{~cm}$

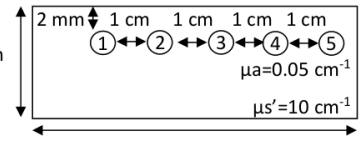

$10 \mathrm{~cm}$ the background fluorescence yield
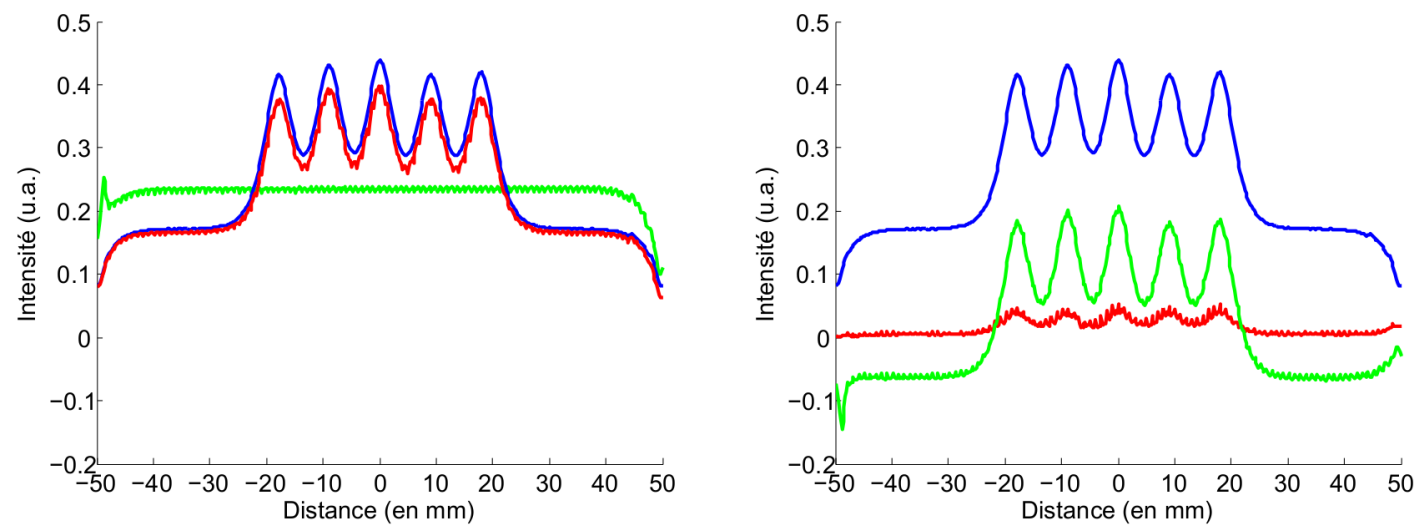


\section{Position 1}

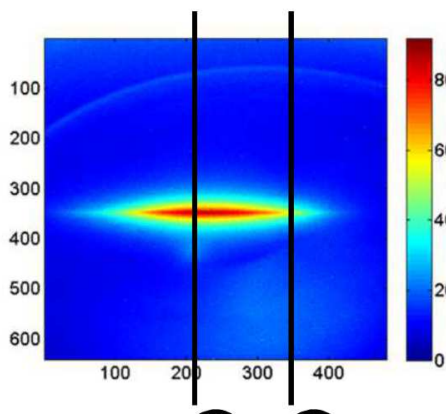

(1) (2)

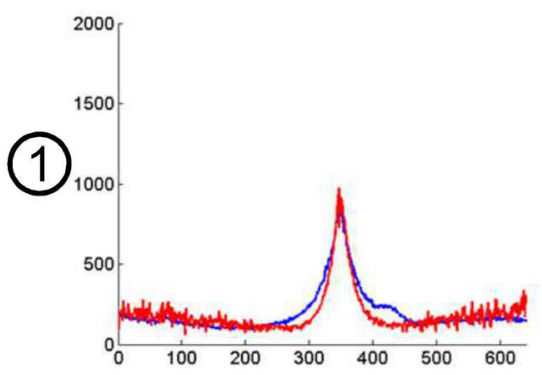

Position 2

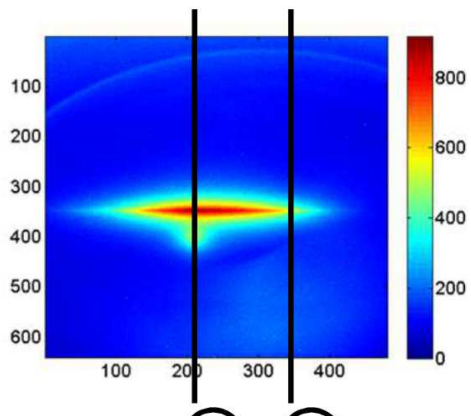

(1) (2)

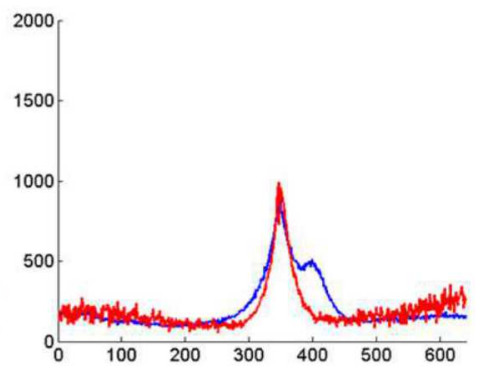

Position 3

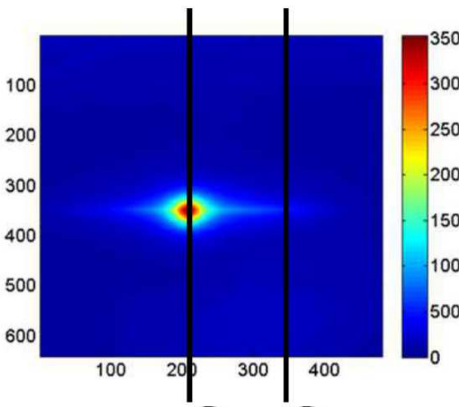

(1) (2)

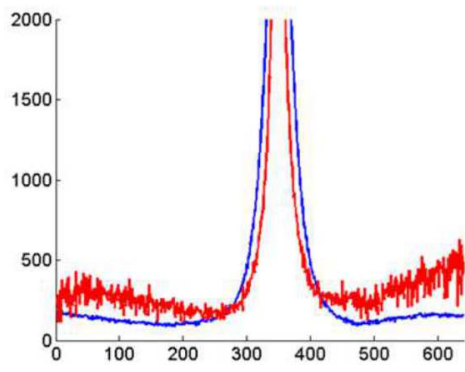

Position 4

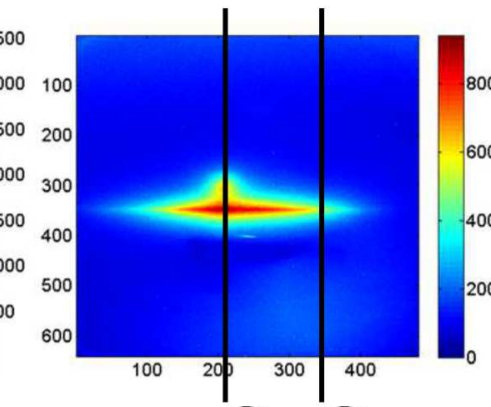

(1) (2)

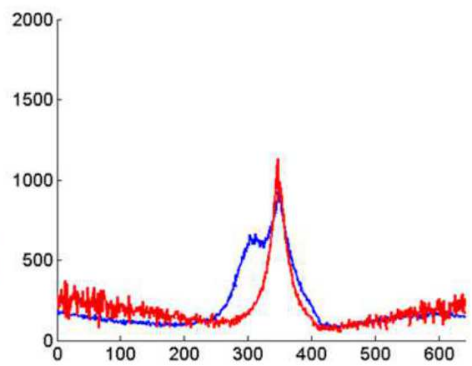

Position 5

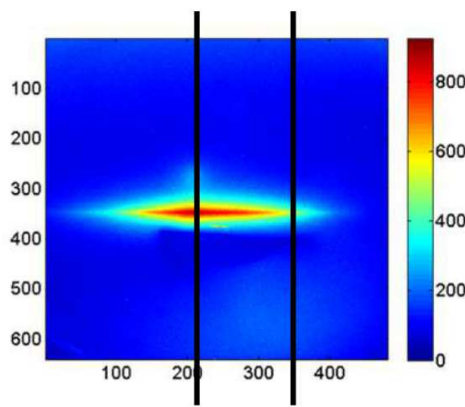

(1) (2)

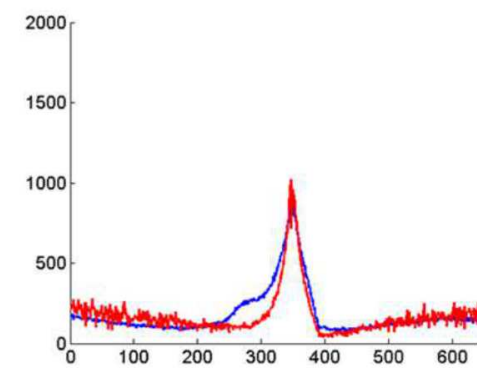

Fluorescence profile (-) and its fit (-) for column 1 at the 5 positions surrounding the inclusion
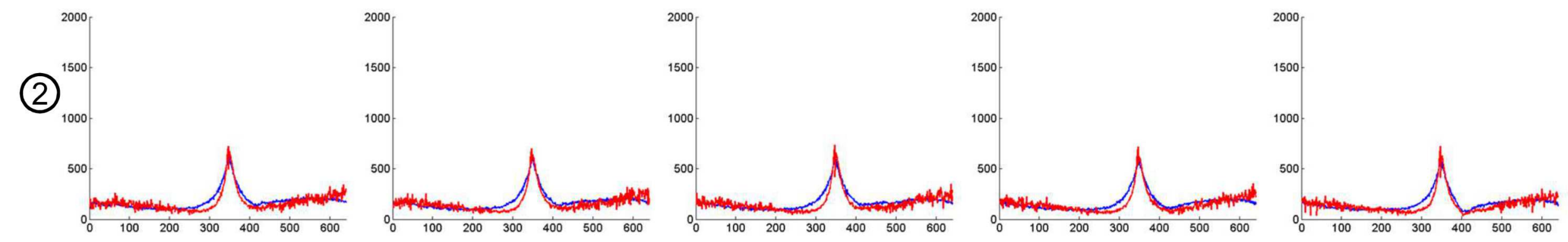

Fluorescence profile (-) and its fit (-) for column 2 at the 5 positions where there is only autofluorescence 

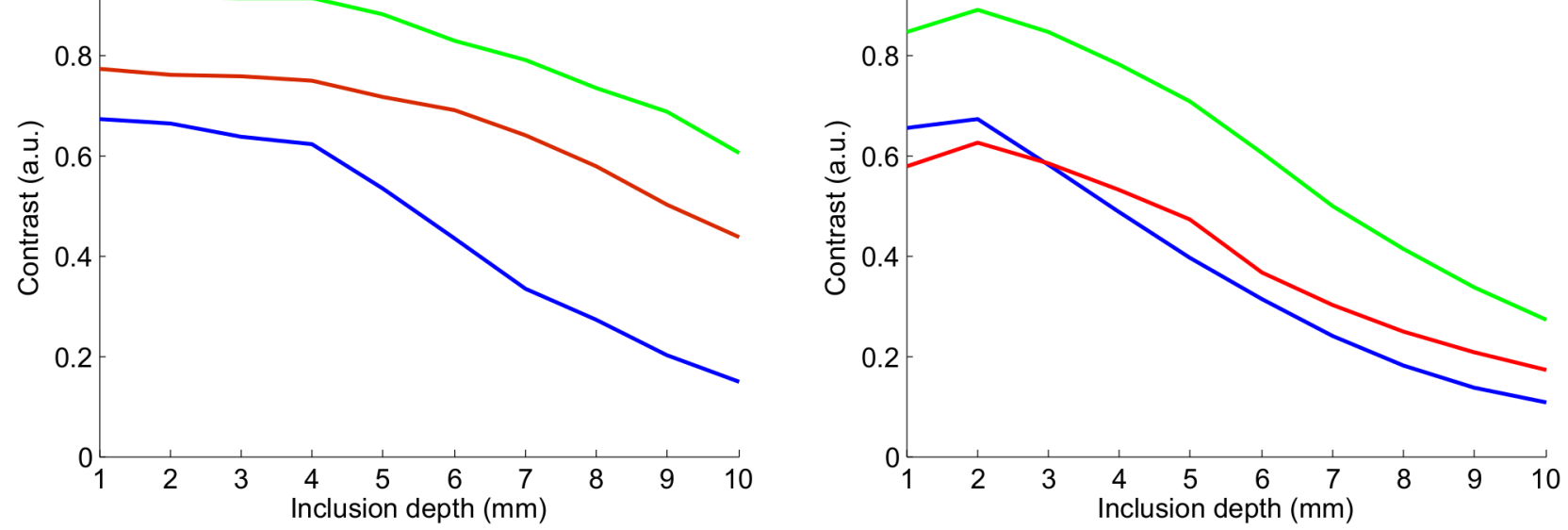


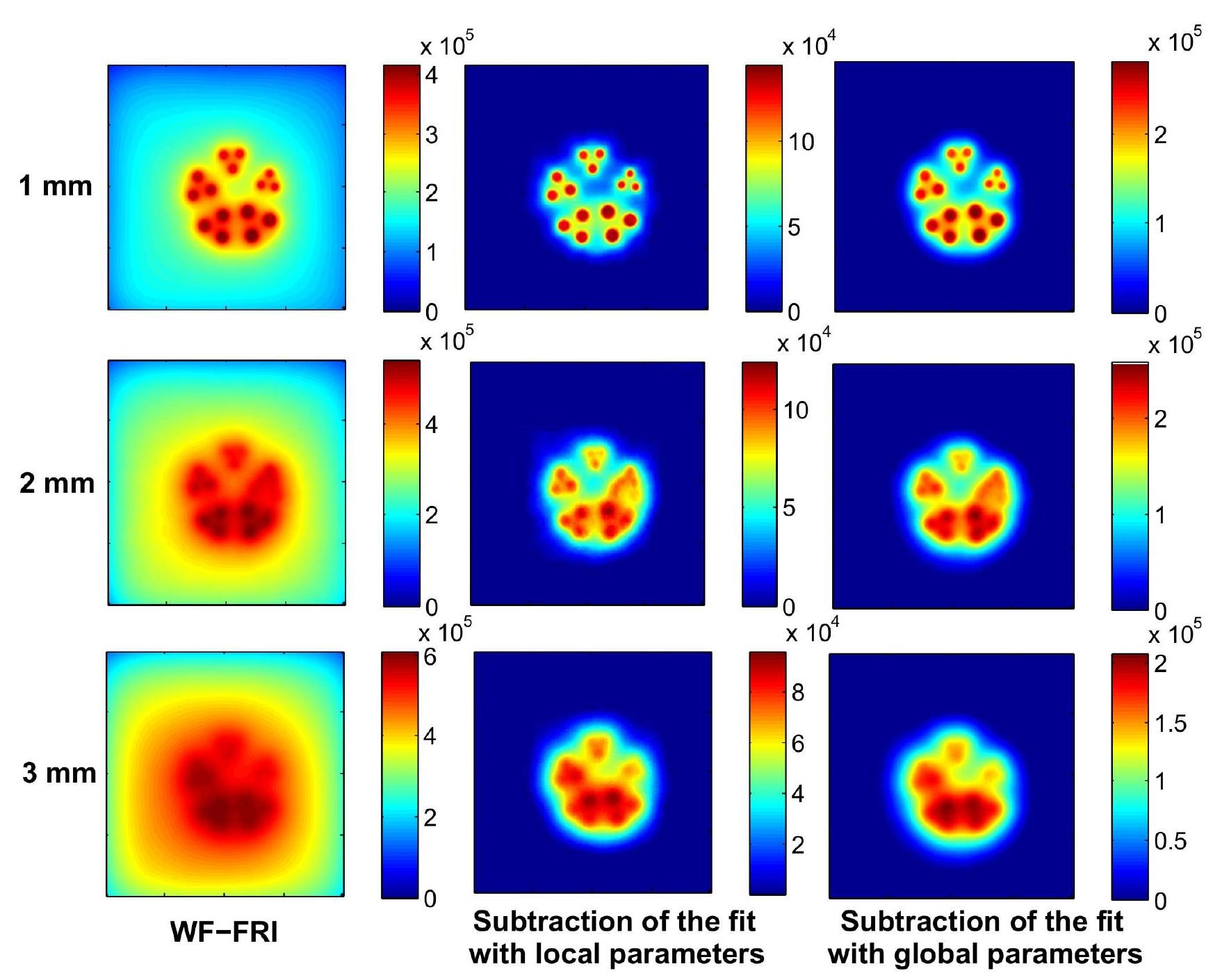


Control without absorption heterogeneity
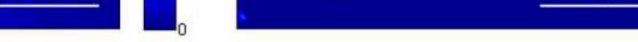

$1 \mathrm{~cm}$

$$
1 \mathrm{~cm}
$$

WF-FRI

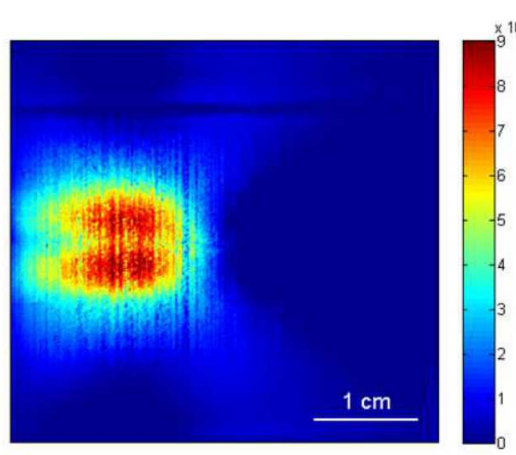

Local parameters fitting method

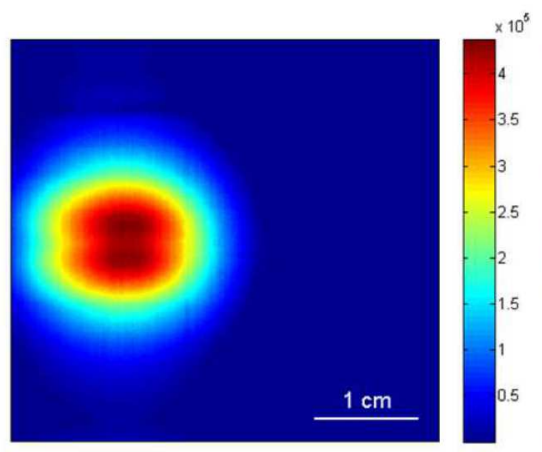

Global parameters fitting method

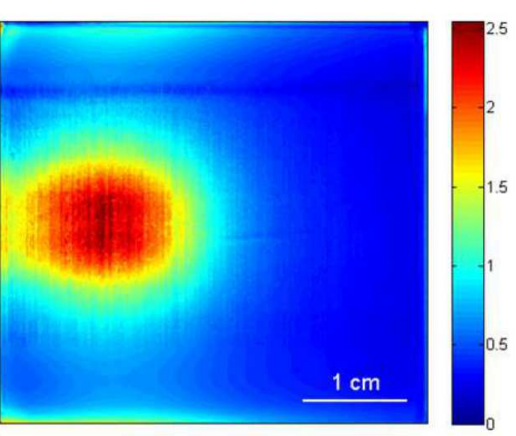

Fluorescence by excitation ratio 


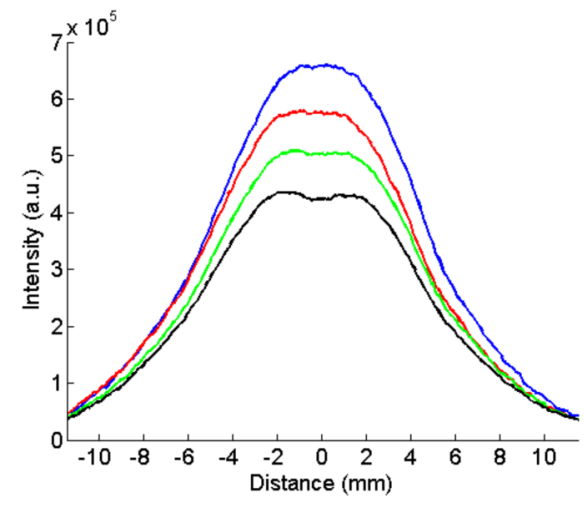

WF-FRI

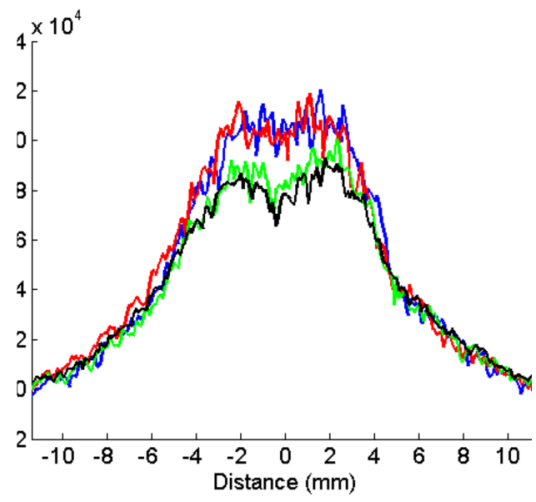

Local parameters fitting method

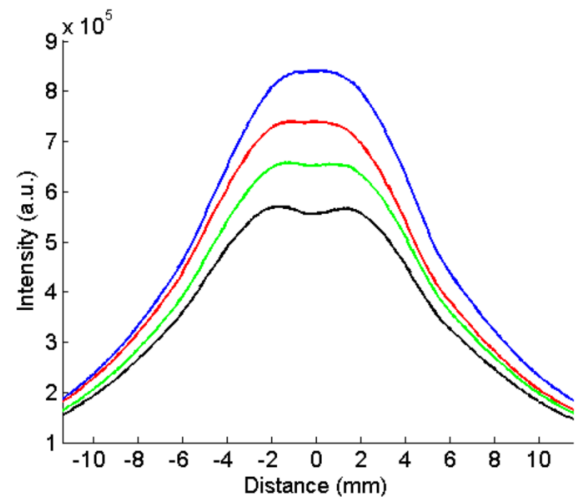

Global parameters fitting method

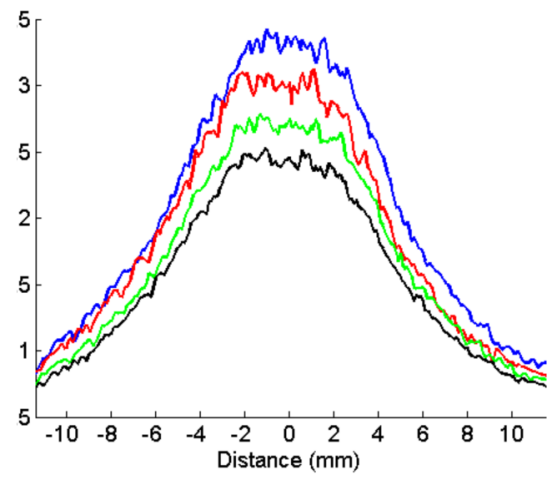

Fluorescence by excitation ratio 
Control with the same absorption coefficients
$1 \mathrm{~cm}$

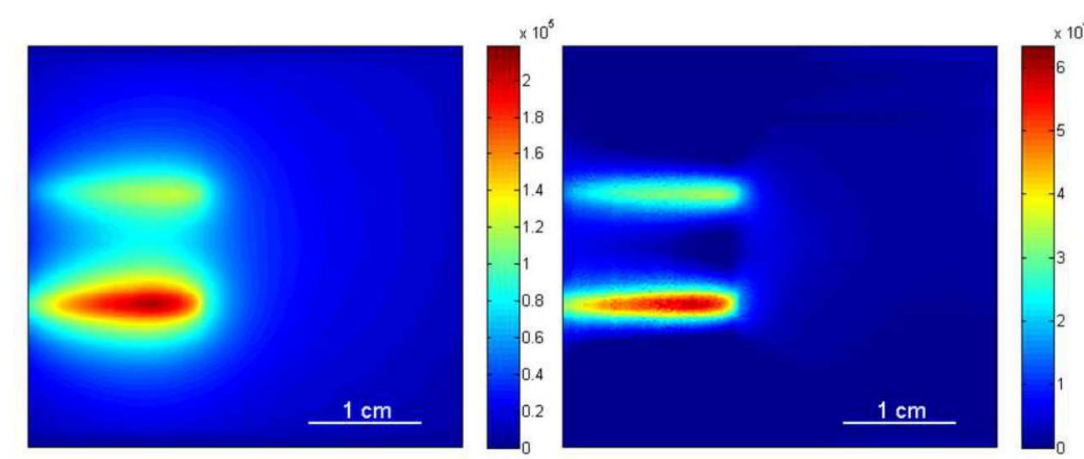

WF-FRI
Local parameters fitting method
$1 \mathrm{~cm}$

$1 \mathrm{~cm}$

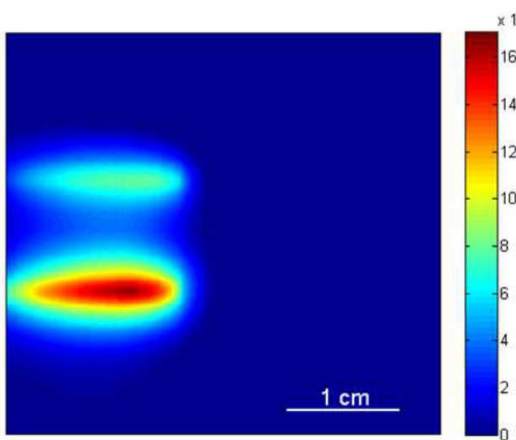

Global parameters fitting method

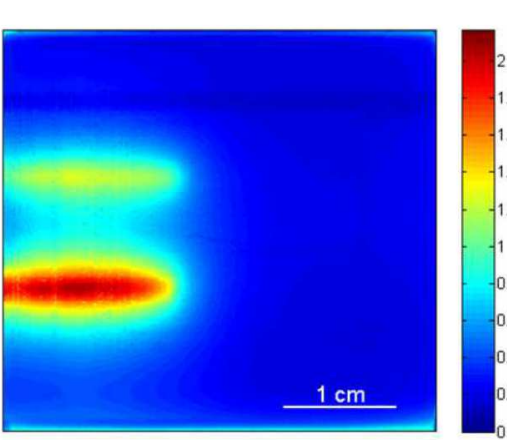

Fluorescence by excitation ratio 


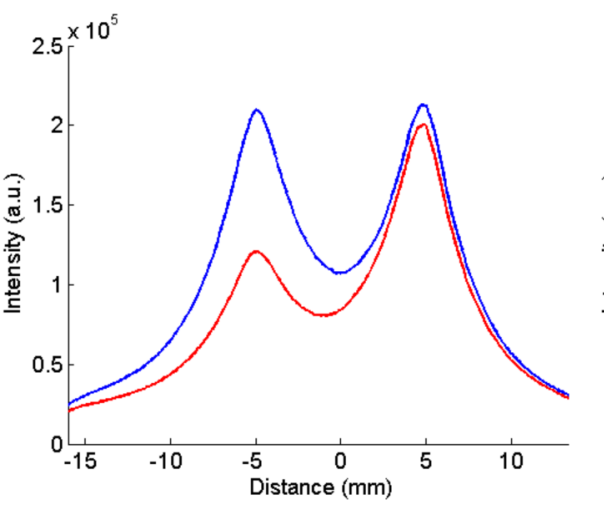

WF-FRI

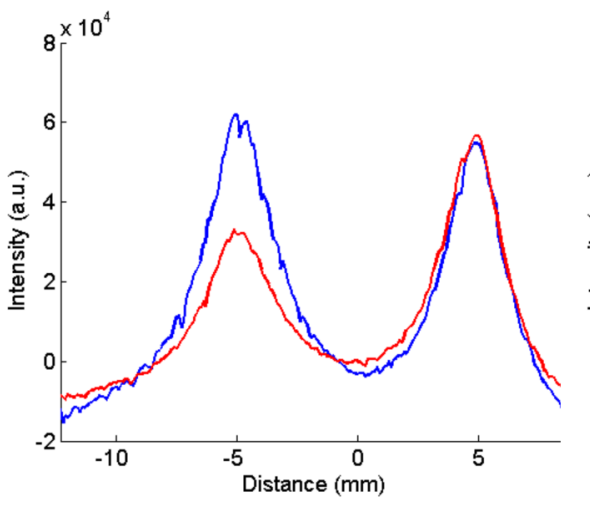

Local parameters fitting method

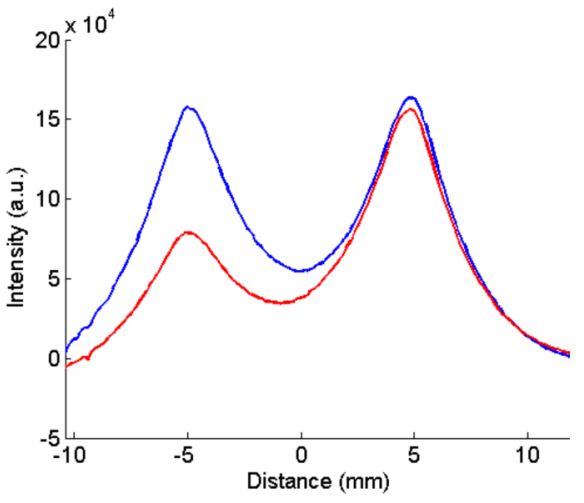

Global parameters fitting method

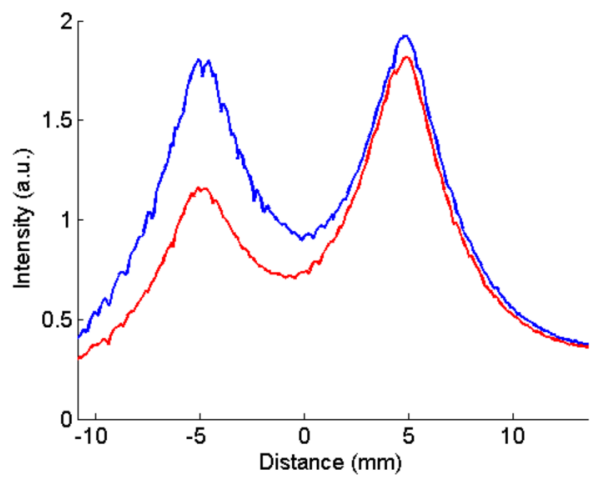

Fluorescence by excitation ratio 